\title{
Exploring the Histone Acetylation Cycle in the Protozoan Model Tetrahymena thermophila
}

\author{
Suzanne Wahab, Alejandro Saettone, Syed Nabeel-Shaht, Nora Dannah and \\ Jeffrey Fillingham*
}

Department of Chemistry and Biology, Ryerson University, Toronto, ON, Canada

\section{OPEN ACCESS}

Edited by:

Christoph Franz Kurat

Ludwig Maximilian University

of Munich, Germany

Reviewed by:

Stephan Hamperl,

Helmholtz Zentrum München,

Germany

Yifan Liu,

University of Michigan, United States

*Correspondence:

Jeffrey Fillingham

jeffrey.fillingham@ryerson.ca

${ }^{\dagger}$ Present address:

Syed Nabeel-Shah,

Donnelly Centre, University of Toronto, Toronto, ON, Canada; Department of Molecular Genetics, University of Toronto, Toronto, ON,

Canada

Specialty section:

This article was submitted to Epigenomics and Epigenetics, a section of the journal Frontiers in Cell and Developmental

Biology

Received: 20 April 2020 Accepted: 28 May 2020

Published: 30 June 2020

Citation:

Wahab S, Saettone A, Nabeel-Shah S, Dannah N and

Fillingham J (2020) Exploring

the Histone Acetylation Cycle in the Protozoan Model Tetrahymena thermophila.

Front. Cell Dev. Biol. 8:509. doi: 10.3389/fcell.2020.00509
The eukaryotic histone acetylation cycle is composed of three classes of proteins, histone acetyltransferases (HATs) that add acetyl groups to lysine amino acids, bromodomain (BRD) containing proteins that are one of the most characterized of several protein domains that recognize acetyl-lysine (Kac) and effect downstream function, and histone deacetylases (HDACs) that catalyze the reverse reaction. Dysfunction of selected proteins of these three classes is associated with human disease such as cancer. Additionally, the HATs, BRDs, and HDACs of fungi and parasitic protozoa present potential drug targets. Despite their importance, the function and mechanisms of HATs, BRDs, and HDACs and how they relate to chromatin remodeling $(\mathrm{CR})$ remain incompletely understood. Tetrahymena thermophila $(\mathrm{Tt})$ provides a highly tractable single-celled free-living protozoan model for studying histone acetylation, featuring a massively acetylated somatic genome, a property that was exploited in the identification of the first nuclear/type A HAT Gon5 in the 1990s. Since then, Tetrahymena remains an under-explored model for the molecular analysis of HATs, BRDs, and HDACs. Studies of HATs, BRDs, and HDACs in Tetrahymena have the potential to reveal the function of HATs and BRDs relevant to both fundamental eukaryotic biology and to the study of disease mechanisms in parasitic protozoa.

Keywords: histone acetyltransferase, bromodomain, chromatin remodeling, histone deacetylase Tetrahymena thermophila, Tetrahymena

\section{INTRODUCTION/BACKGROUND}

\section{Chromatin Remodeling}

Eukaryotic cells package their genomic DNA into chromatin. The basic unit of chromatin, the nucleosome, consists of a histone octamer of four core histones: $\mathrm{H} 2 \mathrm{~A}, \mathrm{H} 2 \mathrm{~B}, \mathrm{H} 3$, and $\mathrm{H} 4$ (Luger et al., 1997). Histone variants are able to substitute for the core canonical histones within the nucleosomes and often confer specific structural and functional features (Henikoff and Smith, 2015). Additional factors, such as linker histones, further organize nucleosomes into higher-order chromatin structures (Fyodorov et al., 2018). Chromatin ultimately needs to be remodeled for DNA transactions such as transcription to occur (Clapier and Cairns, 2009). Mechanisms of chromatin remodeling (CR) involve ATP-dependent histone sliding [e.g., SWI/SNF (Johnson et al., 2005), INO80 (Poli et al., 2017), and ISWI (Yan et al., 2016)] and the selective insertion/removal of histone variants [SWR (Morrison and Shen, 2009) and INO80 (Brahma et al., 2017)], as well as the post-translational modification (Gardner et al., 2011) (PTM) of specific amino acids within histones including lysine acetylation and methylation. 
Histone PTMs can lead to downstream events via recruitment of proteins with specific PTM-interacting domains including the bromodomain (BRD) that interacts with acetylated lysines (Filippakopoulos et al., 2012).

\section{The Histone Acetylation Cycle and Its Relevance to Human Disease}

The histone acetylation cycle begins with the selective addition of an acetyl group to a specific lysine residue in histones, a process known as histone acetylation, and is catalyzed, or "written," by histone acetyltransferases [HATs (Berndsen and Denu, 2008)]. HATs can be guided to their specific histone targets by physically interacting with proteins containing histone-interacting domains (Lalonde et al., 2014). Bromodomain proteins are able to interact, or "read" acetyl-lysine ( $\mathrm{Kac}$ ), and the cycle is complete when the acetyl is removed, or "erased," by histone deacetylases (HDACs) (Kuo and Allis, 1998). Histone acetylation occurs either at the nucleosomal level by type A HATs [SAGA (Baker and Grant, 2007) and NuA4 (Doyon and Cote, 2004) complexes] or on histones prior to their deposition into chromatin by type B HATs [Hatl (Parthun, 2007), Rtt109 (Fillingham et al., 2008)]. Although the focus of this review is histone acetylation, it is important to note that proteomic studies have identified hundreds to thousands of acetylated proteins in a variety of model systems from parasitic protozoa to mammalian cells (Zhao et al., 2010; Jeffers and Sullivan, 2012; Miao et al., 2013; Li et al., 2019). To reflect this, HATs and HDACs that acetylate/deacetylate the group of lysine residues on non-histone substrates are also be referred to as lysine acetyltransferases/deacetylases (KATs/KDACs) (Allis et al., 2007).

The proteins of the histone acetylation cycle have clinical significance in cancer (Somech et al., 2004; Jain and Barton, 2017; Richters and Koehler, 2017) and have attracted interest as potential druggable targets. For example, translocation of BRD-containing BRD4 to NUTM1 in human cells generates an oncoprotein that drives a rare and aggressive form of squamous cell carcinoma, NUT midline carcinoma (NMC) (French et al., 2004). Multiple small molecules have been developed that disrupt BRD-Kac interactions (Cochran et al., 2019) and are subject of investigation for their efficacy in treating cancers such as NMC. HATs, BRDs, and HDACs are often required for the viability and pathogenesis of parasitic protozoa (Jeffers et al., 2017) which are among the Neglected Tropical Diseases (NTD) common in regions of Africa, Asia, and the Americas (World Health Organization, 2010). Anti-protozoa drugs exist but their efficacy is being compromised by rising resistance (de Koning, 2017). The availability of small molecule inhibitors to proteins of the histone acetylation cycle has driven interest in their use to treat these diseases. The Alveolate lineage of eukaryotes includes the parasitic apicomplexa, with Plasmodium and Toxoplasma species. Gcn5 was shown to be essential for Toxoplasma DNA replication, prompting a search for drugs that target this HAT (Vanagas et al., 2012; Jeffers et al., 2016). In Plasmodium, PfBDP1 containing a C-terminal BRD and an N-terminal ankyrin repeat is required for penetration of red blood cells (Josling et al., 2015), potentially providing a drug target. Plasmodium HDACs are also being investigated as possible drug targets (Andrews et al., 2012).

\section{Tetrahymena thermophila as a Model for the Study of the Histone Acetylation Cycle}

The ciliate Tetrahymena thermophila (Tt), a free-living genetically tractable Alveolate, has been a beneficial model for early studies of the fundamental biology of histone acetylation due to its unique nuclear biology (Grunstein and Allis, 2018). Tt is suitable to study apicomplexan biology as well as that of other protozoan parasites due to their evolutionary relationship. Tt is also a proven model for discovery-based chromatin biology, based in part on the biology of the ciliates, which segregate germlinespecific silent, and somatic transcriptionally active chromatin into two distinct nuclei (Orias et al., 2011). The micronucleus (MIC) is diploid, divides by mitosis, and is not transcribed during growth. The MIC undergoes meiosis during conjugation, the sexual phase of the life cycle, and is analogous to a germline nucleus. The macronucleus (MAC) is highly polyploid with $\sim 45$ copies of each MAC chromosome, divides amitotically without functional centromeres, is transcriptionally active, and is not inherited sexually; analogous to a somatic nucleus. During amitosis, multiple copies of each macronuclear chromosome are randomly partitioned between the two daughter cells. As a result, alleles segregate randomly, and therefore vegetative progeny of a cell initially heterozygous after conjugation become homozygous for one of the alleles after a number of cell divisions in a process called phenotypic assortment (Orias and Flacks, 1975). The two nuclei are related to each other through the sexual life cycle, conjugation (Martindale et al., 1982), the milestones of which are shown in Figure 1. MAC development includes a variety of programmed DNA rearrangements that includes chromosome fragmentation, and programmed deletion of DNA sequence called internal eliminated sequences (IESs) which is epigenetically regulated in a process that is initiated by genomewide transcription of non-coding RNAs (Chalker and Yao, 2001) (ncRNAs) from the normally silent MIC. The ncRNAs then direct RNAi-dependent assembly of distinct chromatin domains in the new MAC (Taverna et al., 2002; Liu et al., 2007), a prelude to DNA deletion (Yao et al., 2015) thought to be similar to the chromatin diminution seen in the parasitic nematode Ascaris (Wang et al., 2017).

Tetrahymena thermophila has features of a model genetic organism including fast growth in axenic culture, and the ability to undergo large scale and synchronous matings. Both the MAC (Eisen et al., 2006) and MIC (Hamilton et al., 2016) genomes have been sequenced and annotated (Stover et al., 2006). A well-developed functional proteomic pipeline exists for the study of epigenetic regulators in particular for affinity purification coupled to mass spectrometry (AP-MS) to effectively solubilize native (i.e., un-crosslinked) protein complexes both bound and unbound to chromatin (Xiong et al., 2011; Garg et al., 2013, 2019; Saettone et al., 2018, 2019a; Ashraf et al., 2019; Nabeel-Shah et al., 2020). A critical feature of this approach is the expression of the epitope tagged proteins 


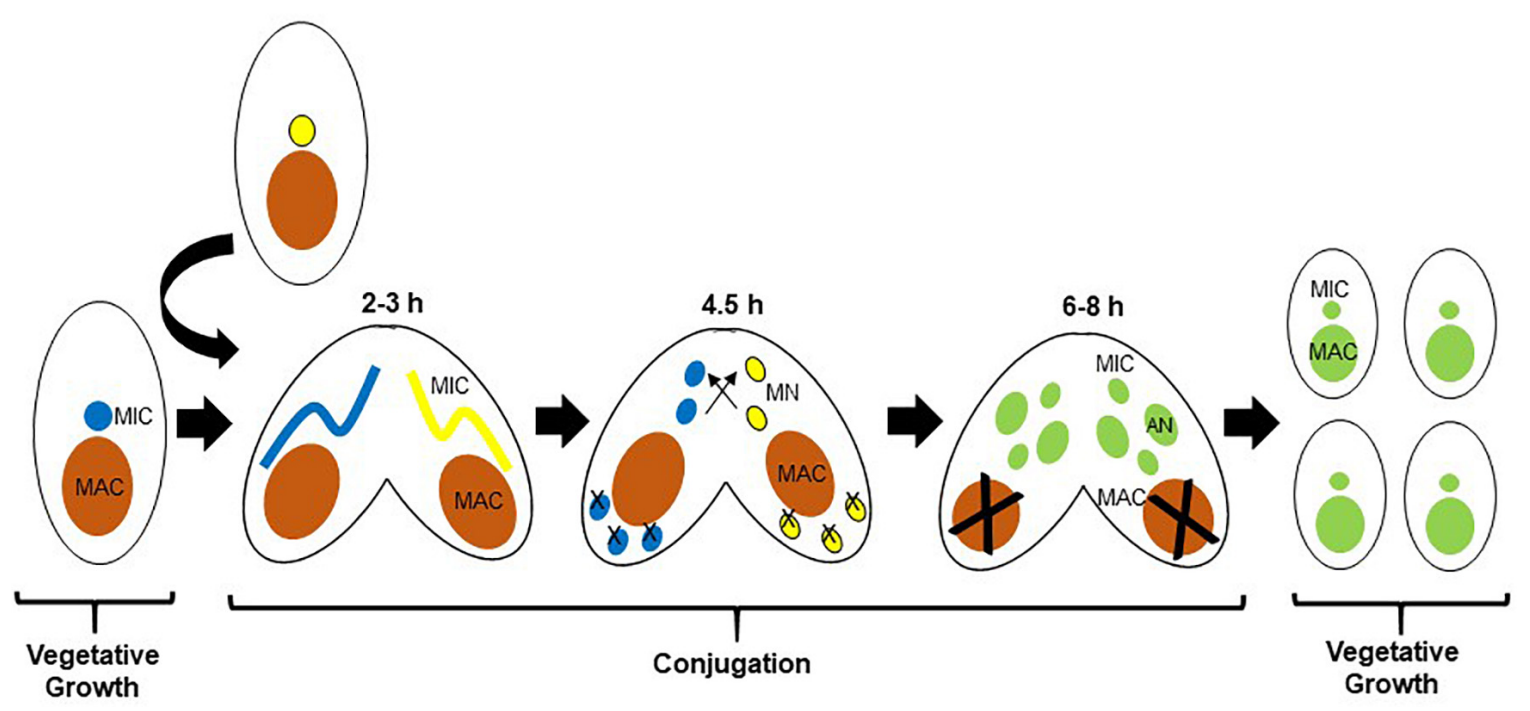

FIGURE 1 | Life cycle of Tetrahymena thermophila (Tt). Parental macronuclei are shown in orange. After pairing of two cells of different mating types, the respective MICs (shown in blue and yellow) undergo meiosis. In each cell, one of the four meiotic products is selected as the nucleus that will be inherited, and the other three degenerate. The selected meiotic product in each cell undergoes mitosis to produce two identical pro-nuclei, one of which is transferred to the mating partner where they fuse to form a zygotic nucleus that further divides mitotically twice to generate four nuclei (shown now in green), two of which will develop as new MACs, and two as MICs. As the new MACs are developing, the parental MAC condenses and degenerates. Time periods shown correspond to hours post mixing of the two cells.

at levels closely approximating that of the endogenous protein. In Tetrahymena this is achieved by exact gene replacement mediated by homologous recombination, adding the epitope tag in-frame at the C-terminus of the endogenous protein, such that the epitope tagged polypeptide is expressed under its own promoter, as in budding yeast. Physical Interactome mapping experiments are performed using a minimum of two biological replicates in parallel to control experiments using untagged parental strains, facilitating the identification of interaction partners significantly over-represented in the samples, a process aided by use of algorithms such as SAINTexpress (Teo et al., 2014). Functional genomic approaches that have been developed for $\mathrm{Tt}$ include definition of the transcriptome through microarray (Miao et al., 2009) and RNA-Seq analysis (Xiong et al., 2011). Because Tt gene expression can be assessed in a variety of developmental stages, network analysis of transcriptome data can be used to predict functional relationships between genes (Xiong et al., 2011). In addition, genomic localization of proteins involved in the acetylation cycle can be tracked using ChIP-Seq (chromatin immunoprecipitation followed by next generation sequencing) (Saettone et al., 2018, 2019b).

\section{HISTONE ACETYLATION IN Tetrahymena thermophila}

In addition to their distinct morphologies and functions, differences exist in the complement of chromatin proteins as well as the degree of histone acetylation in the MAC and MIC. Although the same core histones (H2A, H2B, H3, and $\mathrm{H} 4)$ are present in both nuclei, the MIC possesses two versions of histone H3 (Allis et al., 1979). One of which is indistinguishable from that found in the MAC (named H3S for slow), and the other is unique to MIC, and has a faster mobility in SDS-PAGE (and thus named $\mathrm{H} 3 \mathrm{~F}$ for fast) as a consequence of a regulated proteolytic event where six amino acids are removed from the $\mathrm{N}$-terminus (Allis et al., 1980a; Figure 2A); the underlying enzymology of which is unknown. Nucleus-specific linker histones exist, Hho1 for the MAC and Mlh1 (Micronuclear linker histone 1) for the MIC. Mlh1 in particular is proteolyzed (Allis et al., 1984) into several smaller polypeptides (alpha, beta, gamma, and delta). Although HHO and MLH are non-essential genes, DAPI staining combined with knockout analysis showed that both function in the condensation of chromatin in their respective nuclei (Shen et al., 1995). The MIC also features the exclusive localization of CNA1 (Cervantes et al., 2006; Cui and Gorovsky, 2006), an ortholog of the centromeric-specific H3 variant CENPA, consistent with apparent lack of centromeres in the MAC. Transcription associated histone variants Hv1 (H2A.Z) and Hv2 (H3.3) are MAC-specific (Allis et al., 1980b) during growth and starvation, appearing in the MIC only during selected times in conjugation (Stargell et al., 1993; Cui et al., 2006). In addition to being widely distributed in the MAC by immunofluorescence (IF), Hv1 localizes to the nucleoli indicating that it may be involved in rDNA transcription (Allis et al., 1982). The use of specific anti-Hv1 antibodies in indirect IF indicates that Hv1 may present in the crescent MIC, a time when it is transcriptionally active (Stargell et al., 1993). Hv2 differs in 16 amino acids from the major, abundant $\mathrm{H} 3$ proteins and is expressed constitutively (Thatcher et al., 1994). Genetic analysis suggests that the primary importance of HHT3 in growing cells is a consequence of 


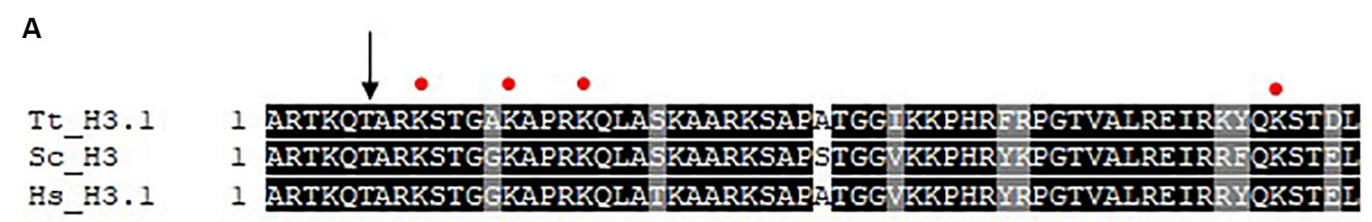

B

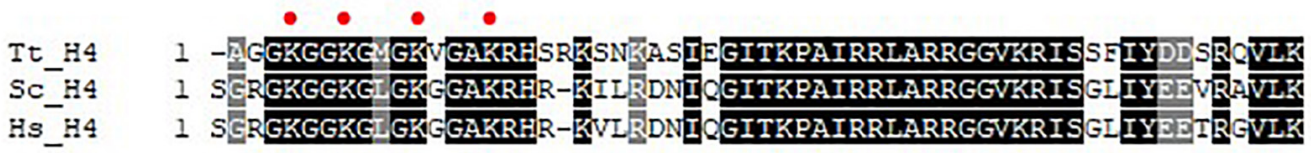

C

Tt_Hvl

$\mathrm{Hs} \mathrm{H} 2 \mathrm{AZ}$

Sc_Htzl

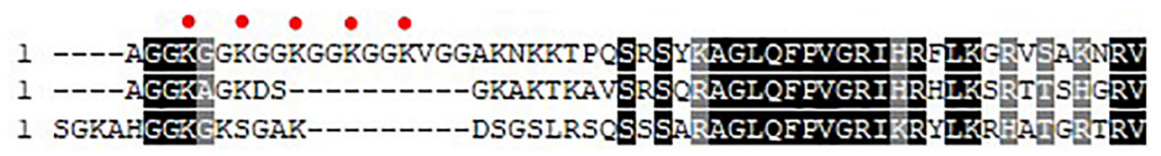

FIGURE 2 | Multiple sequence alignment of histone N-termini. Clustal Omega was used to perform multiple sequence alignment (EMBL-EBI) and shaded using BoxShade (ExPASy). Absolutely conserved residues are represented by a black shade and conserved residues are represented by a gray shade. Red circles represent sites of acetylation as indicated in the text. (A) N-termini of histone $\mathrm{H} 3$. The arrow indicates site of $\mathrm{N}$-terminal proteolysis. (B) $\mathrm{N}$-termini of histone $\mathrm{H} 4$. (C) N-termini of histone H2A variant Hv1/H2AZ. Tt, Tetrahymena thermophila; Hs, homo sapiens; Sc, Saccharomyces cerevisiae.

its constitutive expression rather than its primary sequence (Yu and Gorovsky, 1997).

Some of the earliest observations linking histone acetylation and transcription occurred using the Tt model. Pulse-labeling studies using radiolabeled acetate was used to show that histones in general are highly acetylated in the MAC but not MIC in growing cells (Vavra et al., 1982a,b), a finding that provided a key functional link of histone acetylation to gene expression. Initial studies on histone deposition-associated acetylation focused on pulse-labeling cells with radiolabeled acetate early in conjugation where MICs replicate rapidly, but are transcriptionally inactive, thus any MIC-specific histone acetylation at this time is related functionally to histone deposition and chromatin assembly. Initial studies showed that newly synthesized histone $\mathrm{H} 3$ and histone $\mathrm{H} 4$ were deposited into MIC in mono- and di-acetylated (H3) and di-acetylated (H4) forms (Allis et al., 1985). Later work identified these deposition-associated acetylation sites on K9/14 on H3 (Sobel et al., 1995; Figure 2A) and H4 K4/11 (Chicoine et al., 1986; Figure 2B, note corresponds to H4K5/12 in yeast and human cells: Tt H4 contains a single deletion at amino acid 3, so each acetylation site is numbered one less than that observed in most other H4s). Similar studies on pulselabeled MACs isolated during growth revealed H4K7 (Figure 2B, corresponds to $\mathrm{H} 4 \mathrm{~K} 8$ ) and $\mathrm{H} 4 \mathrm{~K} 4$ to be the principle sites of acetylation correlated with transcription on $\mathrm{H} 4$, with $\mathrm{H} 4 \mathrm{~K} 11$ and H4K15 also acetylated at lower levels (Chicoine et al., 1986; Figure 2A). Transcription-associated sites of acetylation on H3 were revealed to be $\mathrm{H} 3 \mathrm{~K} 9$ and $\mathrm{H} 3 \mathrm{~K} 14$ and to a lesser extent H3K18 and H3K23 (Chicoine et al., 1986; Figure 2B). Subsequent studies found Hv1 to be acetylated at positions 4, 7, 10, 13, 16 in its N-terminus (Allis et al., 1986; Figure 2C). When indirect IF was performed with newly developed anti-tetra-acetylated H4 and anti-penta acetylated Hv1 antibodies, MAC localization was observed (Lin et al., 1989). When studying the extent of histone acetylation during conjugation, only a slight increase in acetylation was shown in the early developing new MACs compared to the developing micronuclei at the same stage. A greater amount of acetylation is detected in the advanced stage of anlagen development comparable to the parental macronuclei (Chicoine and Allis, 1986) suggesting that there is modulation of the histone acetylation cycle during nuclear development.

\section{HISTONE ACETYL-TRANSFERASES IN Tetrahymena thermophila}

Histone acetyltransferases are responsible for catalyzing the transfer of acetyl groups from acetyl-CoA onto lysine residues on the amino-termini of histones. There are five genes that encode clear orthologs of HATs [GCN5/HAT2, HAT1, and 3 MYST-family HATs (MYST1-3)].

\section{Gen5/SAGA}

The search for the gene encoding a HATs was hampered by relatively low amounts of the enzyme that made it difficult to obtain peptide sequence. For example a HAT activity was identified and characterized in yeast in the early 1980s (Travis et al., 1984), but the gene responsible was not cloned (No Author, 2018). The massive amount of acetylated chromatin in the Tt MAC was key to finding the first gene encoding a HATs. A novel SDS-PAGE acetyltransferase activity assay was used to show that the $\mathrm{Tt}$ MAC possess a $55 \mathrm{kDa}$ protein (p55) able to incorporate $[3 \mathrm{H}]$ acetate from [3H] acetylcoA into a histone H3 substrate (Brownell and Allis, 1995). After partial purification using the in gel-assay to monitor the activity, Edman degradation and subsequent molecular 
cloning followed by comparative sequence analysis determined that p55 is orthologous to Gcn5 (Brownell et al., 1996), a transcriptional adaptor, or co-activator previously described as necessary for activity of transcriptional activators in yeast (Georgakopoulos and Thireos, 1992). This finding established the mechanistic link between chromatin structure and gene expression, and reinforced the idea of histone acetylation as a mark of transcriptionally active chromatin. Gcn 5 is broadly conserved in eukaryotes present in most if not all sequenced eukaryotes including Toxoplasma gondii (Wang et al., 2014) and Plasmodium falciparum (Fan et al., 2004). In yeast and human cells, Gcn5 is found in the multi-subunit complex SAGA (Grant et al., 1997), a transcriptional co-activator complex containing $\sim 19$ subunits (Allard et al., 1999; Daniel and Grant, 2007) that, as a type A HAT, targets the nucleosomal N-terminal tail of H3 (Grant et al., 1997, 1998, 1999). The original type A HAT, Gcn5p, may also possess type B HAT activity in Saccharomyces cerevisiae as it is involved in the acetylation of the NH2-terminal tail of newly synthesized histone H3 (Burgess et al., 2010).

Details on the function and mechanism of Tt Gcn5 have lagged since its initial discovery. Recombinant Tt Gcn 5 acetylates free histone $\mathrm{H} 3$ including on $\mathrm{H} 3 \mathrm{~K} 13$ and H3K18 (Garg et al., 2013; Figure 2A). The questions as to the nature of Tt SAGA was recently addressed (Saettone et al., 2018) by AP-MS of Ibd1 (see section "Group II BRD Proteins," below) that revealed p55/Gcn5 to be present in a complex with a clear ortholog of Ada2, as well as the BRD-containing Ibd1, and PHD-domain containing protein $\underline{A}$ da2-Associated Protein $\underline{1}$ (AAP1). One function of PHD domains is to interact with methyl-lysine PTMs (Arrowsmith and Schapira, 2019). Subsequent AP-MS of Ada2 reciprocally identified Gcn5, Ibd1, Aap1 and three additional PHD domain-containing proteins (Aap2-4) that were not observed in repeated Ibd1 AP-MS. Consistent with this, a SAGAlike complex consisting of orthologs of Gcn5, Ada2, and a PHD domain protein (PHD1) in addition to a protein of unknown function, was purified from Apicomplexan $P$. falciparum by incubation of extracts with a biotinylated H3K4me3 peptide (Hoeijmakers et al., 2019). The same complex minus the protein of unknown function co-purified when extracts were incubated with an $\mathrm{H} 4 \mathrm{~K} 5 / 8 / 12 \mathrm{ac}$ peptide. Additionally, AP-MS of PfGcn5 revealed an interaction with an additional PHD-domaincontaining protein PHD2, purification of which enriched Gcn5 and Ada2 but not PHD1. The similarities between Gcn5 membership in multiple protein complexes in Tt and Pf suggest that multiple SAGA-like complexes exist in Alveolates that are composed of a "core" of Ada 2 and Gcn 5 with different epigenetic readers that in $\mathrm{Tt}$ and $\mathrm{Pf}$ are composed of $\mathrm{PHD}$ and $\mathrm{BRD}$ proteins. To demonstrate this conclusively, AP-MS of Tt Aap2-4 will be required, the prediction being that each will co-purify with Ada2 and Gcn 5 but none of the other readers. If this is the case, it will be important to determine chromatin-binding specificity of the respective PHD fingers of the AAPs. BLASTP analysis indicates that AAP1 is the highest match in the $\mathrm{Tt}$ genome of Pf PHD1 with high homology between the two in their fourth PHD domain of PHD1 that matches consensus for H3K4me2/3-binding.

\section{Hat1}

Hat1 was originally purified from yeast cytoplasmic extracts in a complex with Hat2 (Rbap46 in mammalian cells). Hat1 is a type B HAT, highly specific for H4K5 and H4K12 on free histones in yeast and humans (Parthun, 2007). Tt encodes a clear ortholog of HAT1, but to date it has not been subjected to molecular analysis. Experiments performed by Allis and colleagues showed MIC and cytoplasmic extracts of growing Tt to possess a HAT activity on Tt H4 (Richman et al., 1988) that had specificity for position 4 or positions 4 and 11 (Richman et al., 1988; Figure 2B, the sites that correspond to $\mathrm{H} 4 \mathrm{~K} 5 / 12$ in $\mathrm{Tt}$ ). The same activity did not acetylate mononucleosomes, consistent with Hat1 activity in yeast and human cells (Parthun et al., 1996; Verreault et al., 1998). Based on work performed in yeast and human cells, it would make sense that this activity was performed by the Hat 1 complex (Parthun et al., 1996), composed of the Hat1 HAT bound to the Hat2/RbAp46 histone chaperone (Parthun, 2013). The expression of HAT1 is essential in human cells (Nagarajan et al., 2013). Interestingly, when the cytoplasmic activity was heated to $45^{\circ} \mathrm{C}$, the Hat1-like activity on $\mathrm{H} 4$ was retained, but now the activity also mono-acetylated $\mathrm{H} 3$ at an unknown lysine residue (Richman et al., 1988). It will informative to characterize the Hat1 complex in $\mathrm{Tt}$ and to determine whether it has activity on $\mathrm{H} 3$ as well as $\mathrm{H} 4$.

\section{NuA4/Esa1}

NuA4/TIP60 are multi-subunit type A HAT complexes in yeast and human cells responsible for acetylation of nucleosomal histone $\mathrm{H} 4$ and $\mathrm{H} 2 \mathrm{~A}$ with catalytic subunit Esa1/Tip60 (Doyon and Cote, 2004; Jacquet et al., 2016). Analogous H2A/H4specific Hat A activities are more poorly characterized outside the Opisthokonts. In $\mathrm{Tt}$, a $\mathrm{H} 2 \mathrm{~A} / \mathrm{H} 4$-specific nucleosomal HAT activity was previously partially purified from MAC DNAsetreated extracts, and labeled NuA4-like (Ohba et al., 1999). Yeast/human NuA4/TIP60 complexes and the Tt NuA4-like activity both have specificity for lysines $5,8,12$, and 16 of $\mathrm{H} 4$ and lysines 5 and 9 of H2A on nucleosomes (Ohba et al., 1999), suggesting the activity could be catalyzed by an analogous $\mathrm{Tt}$ complex. However, the Tt NuA4-like activity co-purified on a sucrose gradient with a predicted size of $\sim 80 \mathrm{kD}$ which is much smaller than that of NuA4/TIP60 complex, a 1.0-1.5 MDa multi-protein platform of at least 13-16 subunits. Comparative sequence analysis of the MAC genome suggests that there are three potential $\mathrm{Tt}$ genes encoding an ortholog of Esa1/Tip60, named MYST1-3, with MYST1 and MYST2 situated side by side in the MAC genome, possibly the result of a tandem duplication. Yeast also encode three MYST family HATs, each of which nucleates a distinct HAT complex with non-overlapping functions (Esa1-NuA4, Sas2 - SAS complex and Sas3 of the NuA3 complex). The parasitic protozoa Trypanosoma brucei (T. brucei) encodes three MYST family proteins named HAT1-3 that all localize to its nucleus (Kawahara et al., 2008). P. falciparum encodes a single gene encoding a MYST family HAT (Miao et al., 2010). Clear orthologs of genes encoding core NuA4/Tip60 proteins Eafl and Epl1 are not present in the Tt MAC genome or that of other Alveolates, consistent with the idea that a NuA4 complex is not well conserved outside the Opisthokonts. 
Identification of the Tt MYST HAT underlying the NuA4-like activity, and identification of co-purifying proteins, is likely to inform NuA4/TIP60 characterization in the parasitic protozoa.

\section{H3K56 Acetylation in Tetrahymena thermophila}

$\mathrm{H} 3 \mathrm{~K} 56 \mathrm{ac}$ is associated with DNA replication associated chromatin assembly, gene expression, and maintenance of genome stability in yeast and human cells (Masumoto et al., 2005; Das et al., 2009). H3K56 is conserved in Tt H3 (Figure 2A), and the MAC possesses robust levels of $\mathrm{H} 3 \mathrm{~K} 56 \mathrm{ac}$ during growth (Garcia et al., 2007) and early nuclear development (Akematsu et al., 2017). Although not widely studied outside of Fungi and human cells, H3K56ac has been reported to be present in parasitic protozoa such as P. falciparum (Gupta et al., 2016). H3K56ac is catalyzed by Rtt109 in Fungi (Collins et al., 2007; Driscoll et al., 2007; Fillingham et al., 2008) and p300/CBP in humans (Das et al., 2009). In Fungi and human cells, histone $\mathrm{H} 3 / \mathrm{H} 4$ chaperone ASF1 is also required to catalyze H3K56ac (Recht et al., 2006; Das et al., 2009) by the respective HAT. Tt does not encode a clear ortholog of either Rtt109 or p300/CBP but does encode a single copy of ASF1 (Garg et al., 2013). Rtt109 has drawn recent interest as a possible drug target to combat pathogenic fungal infection due to its fungal-specific nature and importance to viability (Wurtele et al., 2010). Despite their non-homologous primary amino acid sequence, Rtt109 and p300/CBP have structural similarity of their catalytic core (Bazan, 2008). Human Gcn5 has been reported to acetylate $\mathrm{H} 3 \mathrm{~K} 56$ in human cells (Tjeertes et al., 2009), but not in yeast. Recombinant Tt p55/Gcn5 does not possess $\mathrm{H} 3 \mathrm{~K} 56 \mathrm{ac}$ activity in vitro on core histone substrates in the presence or absence of recombinant yeast or Tt Asf1 (Garg et al., 2013) which argues against Gcn5 being the H3K56specific HAT in Tt. It should be noted that the HAT assay was performed with chicken and not $\mathrm{Tt}$ histones, and that in $\mathrm{Tt}$ Gcn5 exists in a protein complex with an Ada2 ortholog (see section "Gcn5/SAGA"), so its behavior in vitro may reflect absence of key components. Functionally, the importance of the modification to growth/genome stability in $\mathrm{Tt}$ has yet to be determined, or even if it is linked to chromatin assembly as in yeast. Arabidopsis have also been demonstrated to encode orthologs of p300/CBP suggesting that p300/CBP was present in last common ancestor of plants and Opisthokonts, which should also include protist lineages. If $\mathrm{H} 3 \mathrm{~K} 56 \mathrm{ac}$ is important to protozoan viability, the H3K56-specific HAT (particularly if novel) may have potential as a drug target for treatment of parasitic protozoa infection.

\section{BROMODOMAIN PROTEINS IN Tetrahymena thermophila}

There are several protein domains that selectively recognize and bind to acetylated Lysine (Kac) residues in histones (Jain and Barton, 2017) including PHD (Zeng et al., 2010), YEATS (Li et al., 2014) and the BRD that is the focus of this section. BRD-containing proteins are frequently dysregulated in cancer (Jain and Barton, 2017) and their expression has been demonstrated to be important for pathogenesis of several parasitic protozoa (Schulz et al., 2015). Importantly, BRDs can be targeted by small-molecule inhibitors leading to the idea they can be targeted to control cancer and/or infection by parasitic protozoa (Jeffers et al., 2017; Kougnassoukou Tchara et al., 2019; Hanquier et al., 2020; Nguyen et al., 2020). The polyploid MAC of $\mathrm{Tt}$ is massively enriched for acetylated chromatin which makes it a potentially useful system for discovering new BRD functions. A BLASTP-based survey of the Tt MAC genome revealed 14 potential BRD-containing gene products (Saettone et al., 2018). Tt BRD proteins present one BRD per protein which is different to other described eukaryotes where BRD proteins often are present in tandem. The BRD is composed of four helices with the ZA and BC loops connecting helices $\alpha Z$ to $\alpha A$, and $\alpha B$ to $\alpha C$. The ZA and BC loops form a hydrophobic pocket that functions in Kac binding (Dhalluin et al., 1999). The ZA and BC loops interact with residues flanking the Kac and are somewhat variable in sequence, reflecting the fact that different BRDs have distinct lysine acetylation sites in histones and non-histone proteins (Zaware and Zhou, 2019). A structure-based multiple sequence alignment of the $14 \mathrm{Tt}$ BRDs (Figure 3) shows conservation of BRD secondary structure. BRD-based Kac recognition involves hydrogen bond formation with a conserved Asparagine in the BC loop (Zaware and Zhou, 2019) which is conserved in all $14 \mathrm{Tt}$ BRD proteins (Figure 3). Phylogenetic analysis was performed using the 14 Tt BRD sequences (Saettone et al., 2018) permitting their delineation into three main groups which is shown along with domain analysis of the respective full length protein in Figure 4.

\section{Group I BRD Proteins}

Group I is composed of two proteins, the first a clear ortholog of Mixed-Lineage Leukemia 1 (MLL1), and BroP3 (Figure 4). Mammalian MLL1 is a histone methyl-transferase (KMT2A) that positively regulates transcription by tri-methylation of $\mathrm{H} 3 \mathrm{~K} 4$ (H3K4me3) and is considered an ortholog of yeast Set1. Both mammalian MLL1 and yeast Set1 co-purify with a related protein complex, COMPASS (Shilatifard, 2012). A previous study demonstrated that hyper-acetylated MACspecific histone $\mathrm{H} 3$ tends to be rich in $\mathrm{H} 3 \mathrm{~K} 4 \mathrm{me} 3$ (Taverna et al., 2007). It seems possible that in Tt, highly expressed genes are rich in both modifications. Our sequence alignment indicates that the BRD of Tt MLL1 [unlike that of human (Filippakopoulos et al., 2012)] is predicted to have Kac-binding activity (Figure 3) but its relatively divergent ZA loop sequence (Figure 3) raises the possibility of a non-histone target. It is tempting to speculate that the BRD of MLL1 recruits histone methyltransferases (HMTs) to add the H3K4me3 modification to acetylated chromatin, promoting high levels of transcription. Interestingly, GroupII BRD protein Ibd1 co-purifies with another putative H3K4me3 HMT that does not itself possess a BRD (see below Section "Group II BRD Proteins"). BroP3, the other group I member, has a simple domain structure similar to Ibd1 (Section "Group II BRD Proteins") with a single N-terminal BRD. 


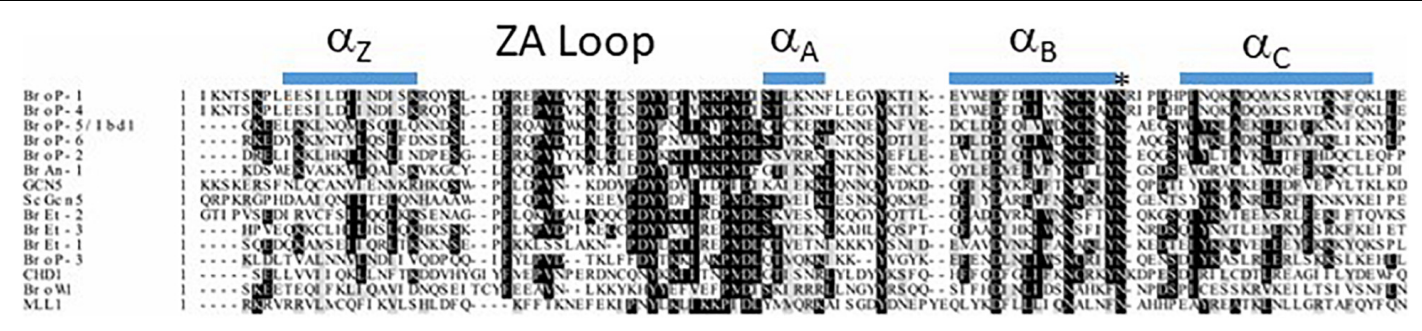

FIGURE 3 | Multiple sequence alignment of 14 Tt bromodomains (BRD). BRD sequence was extracted from the respective full length sequence using SMART. Clustal Omega was used to perform multiple sequence alignment (EMBL-EBI) and shaded using BoxShade (ExPASy) with absolutely conserved residues indicated by a black shade and conserved residues by a gray shade. The asterisk represents the highly conserved Asparagine ( $\mathrm{N}$ ) that makes contact with acetyl-lysine. Predicted secondary structure (JPRED) is shown along the top of the alignment.

\section{Group II BRD Proteins}

Group II is subdivided into two principal divisions (Figure 4). Group IIA includes chromodomain helicase protein (CHD), with BRD situated between two N-terminal chromodomains, in addition to BroW1, possessing several WD-repeats and a C-terminal BRD. The MIC copy of the gene encoding BroW1 includes an IES (Hamilton et al., 2016). IES are rarely found in MIC sequence corresponding to coding sequence in the MAC due to their imprecise mechanism of elimination.

Group IIB includes BrAn1, in addition to several proteins related by their simple domain organization, BroP1, BroP2, BroP4, BroP5, and BroP6 (Figure 4). BroP5 (also known as Ibd1 -Interactive $\mathbf{B R D}$ protein $\mathbf{1}$ ) is the best characterized $\mathrm{BRD}$ protein to date in Tt, initially identified as a member of the Tt SWI/SNF CR complex via AP-MS of the conserved Snf5 subunit (Saettone et al., 2018). Reciprocal AP-MS experiments revealed Ibd1 to also be a member of SWR, a SAGA-like and an HMT-containing complex (Saettone et al., 2018). Incubation of recombinant Ibd1 with a histone post-translation modification peptide array (Saettone et al., 2018) suggest that its BRD recognizes specific histone acetylation associated with the transcriptionally active MAC, H3K9/14ac, and H4K8ac (Chicoine et al., 1987; Taverna et al., 2007). Indirect IF of Ibd1 showed localization to the MAC throughout the Tt life cycle consistent with a role for Ibd1-containing CR complexes in the regulation of transcription (Saettone et al., 2018). Support for this hypothesis was provided by ChIP-Seq analysis of Ibd1 that revealed enrichment in the coding regions of highly expressed genes during growth leading to the proposal of the "pile-on" model where histone acetylation leads to Ibd1-dependent recruitment (or "piling on") of multiple CR activities that each individually contribute to high levels of transcription. To test the model, it will be important to assess the degree to which Ibd1 recruits each of the respective CR complex to the set of Ibd1-bound highly expressed genes. Also, although the model proposes an additive effect for each CR complex, it is possible that redundancies exist among them. The CR complexes hypothesized to be recruited by the Kac-binding of Ibd1 BRD include SWR, SWI/SNF, SAGA and a putative HMT.

The putative HMT that co-purifies with Ibd1 is uncharacterized, but sequence analysis indicates that it has homology to HMTs that have specificity for H3K4 or H3K36, both modifications associated with transcription. As discussed above for MLL, the association of Ibd1 and the HMT potentially could link transcription-associated histone acetylation to H3K4me3, providing a molecular mechanism behind the observation that hyper-acetylated MAC-specific histone $\mathrm{H} 3$ tends to be rich in H3K4me3 (Taverna et al., 2007). The SWI/SNF complex is an ATP-dependent CR complex nucleated by SNF2/Brg1 ATPase subunit. The human Brd9 protein, similar to Ibd1 with only one BRD, was recently shown to be a member of human SWI/SNF complex (Wang et al., 2019). Unlike yeast and human cells, Tt Brg1/SNF2 does not possess a C-terminal BRD (Fillingham et al., 2006). The specificity of the Ibd1 BRD appears similar to that of the human and yeast $\mathrm{Brg} 1 / \mathrm{Snf} 2$ subunit. The biochemical function of SWI/SNF is ATP-dependent remodeling of nucleosome structure by mobilizing nucleosomes by sliding and/or ejection of histone octamers (Saha et al., 2006). We speculate that the physical interaction of Ibd1 with SWI/SNF links histone acetylation to nucleosome sliding or ejection. Interestingly the association between Ibd1 and SWI/SNF appears much less stable during conjugation (Saettone et al., 2018) indicating that the physical interaction between the two may be subject to regulation. The function of SWR-C in yeast (SRCAP in humans) is the deposition of Htz1/H2AZ (Kobor et al., 2004; Ruhl et al., 2006). Previously the Allis lab demonstrated that Hv1, like Ibd1, localizes to the MAC of growing cells implicating Hv1 in transcription (Wenkert and Allis, 1984). Tt SWR-C was defined by the intersection of common proteins in Ibd1 and Swc4 AP-MS (Saettone et al., 2018; Ashraf et al., 2019) with clear orthologs identified for Swr1, Yaf9, Rvb1, RvB2, Swc2, Swc4, and Swc5. AP-MS of Hv1 co-purified Swr1, Swc2, Arp5, and Rvb1 (Ashraf et al., 2019), consistent with Tt SWR-C function in the deposition of Hv1. In yeast, the NuA4 HAT acetylates H4 which recruits SWR via the BRD-containing Bdf1 subunit to deposit Htzl (Durant and Pugh, 2007; Altaf et al., 2010). It is tempting to speculate that the Tt NuA4-like activity (discussed above) acetylates $\mathrm{H} 4$, proving a platform for Ibd1 recruitment and subsequent Hv1 deposition. Support for this model rests in the fact that Ibd1 appears to recognize H4K8ac (Saettone et al., 2018) [analogous to H4K7ac (Figure 2B)] that is characteristic of NuA4 activity (Allard et al., 1999).

Group IIB proteins also include BroP6, BroP2, and BroP4, that are similar to BroP5/Ibd1 in that they are small ( $\sim 400 \mathrm{aa})$, with 


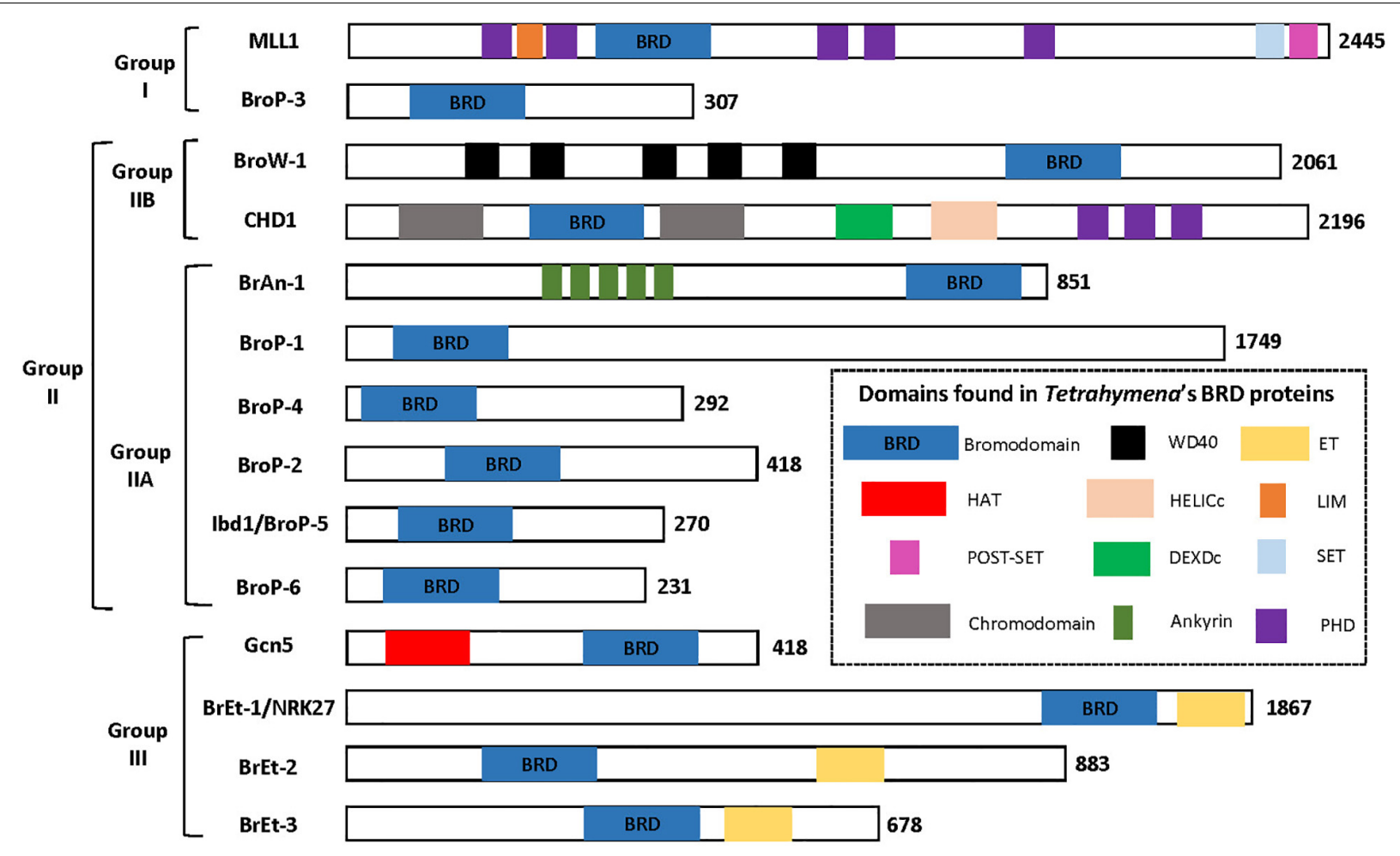

FIGURE 4 | Tetrahymena's 14 BRD proteins grouped based on phylogenetic analysis of BRD sequence. Figure adapted from Saettone et al. (2018).

a single N-terminal BRD and no other recognizable domains. BroP6 in particular is highly similar to Ibd1/BroP5 in amino acid sequence and along with Ibd1 co-purifies in Hv1 AP-MS (Ashraf et al., 2019). INO80-C is a multi-subunit ATP-dependent CR complex (Poli et al., 2017) that possesses a histone-exchange activity that swaps nucleosomal H2A.Z/H2B with $\mathrm{H} 2 \mathrm{~A} / \mathrm{H} 2 \mathrm{~B}$ (Papamichos-Chronakis et al., 2011) (in essence, the opposite of SWR-C). Several potential INO80-C subunits co-purified in AP-MS of Hv1-FZZ (Ashraf et al., 2019) including seven evolutionary conserved subunits present in INO80-C in yeast and human including INO80, Arp5, Arp8, and IES6 in addition to transcription factor YY1, a FHA domain protein and a ubiquitin hydrolase all present in human but not in yeast INO80-C. INO80$\mathrm{C}$ has not been reported to possess a BRD protein in yeast but in human cells can be co-purified with Brd3 (Wai et al., 2018). Although a definitive physical link between Brop6 and Tt INO80-C awaits AP-MS of BroP6, it is tempting to speculate that while Ibd1 recruits SWR-C to deposit Hv1 into chromatin via acetylation of $\mathrm{H} 4$, BroP6 could regulate its removal via initial recognition of a different acetyl modification. If BroP6 is in fact found to regulate Hv1 removal, it will be important to determine the basis of Hvl recognition. One possibility is that N-terminal Hv1 acetylation itself could be recognized by a BRD, although a previous genetic study argues that the essential function of Hv1 acetylation is mediated through a charge patch, not a trans-acting factor (Ren and Gorovsky, 2001).

The only BRD reported to directly recognize acetyl-H2AZ is T. brucei BDF2 (Yang et al., 2017). The more likely possibility is that $\mathrm{Hv} 1$ is recognized within an Kac nucleosomal context, analogous to how human BRD2 recognizes H2AZ-containing nucleosomes containing acetyl-H4 (Draker et al., 2012). The Allis lab used an affinity purified polyclonal anti-Hv1 antibody to argue that Hv1 (unlike Ibd1) is be present in the meiotic MIC and may function in the pathway of MIC-specific genomewide ncRNA transcription (Martindale et al., 1985; Stargell et al., 1993). Further work will be necessary to uncover the Ibd1indepdent mechanism by which Hv1 is deposited in the meiotic MIC, but because of the apparent absence of Ibd1, it is unlikely to be related to histone acetylation.

The last Group IIB protein BrAn1 has a domain architecture consisting of a C-terminal BRD in combination with several $\mathrm{N}$-terminal ankyrin repeats, resembling that of PfBDP1 in P. falciparum, the knockdown of which reduced ability to penetrate red blood cells with concomitant deregulation of invasion-associated genes (Josling et al., 2015). Interactome analysis of PfBDP1 indicates that it forms a core complex with an additional BRD protein, PfBDP2 (Hoeijmakers et al., 2019) that appears to form a variety of sub-complexes with additional proteins including a PHD-domain containing protein (Hoeijmakers et al., 2019) and a DNA-binding transcription factor AP2-I (Santos et al., 2017). The domain architecture of PfBDP1, and Tt BrAn1 is also conserved in the T. gondii ortholog (TGME49_263580) and appears to be unique to select protist lineages including $\mathrm{Tt}$ and apicomplexans (Jeffers et al., 2017). Functional characterization of $\mathrm{Tt} \mathrm{BrAn} 1$ should contribute to the understanding of the apicomplexan version. 


\section{Group III BRD Proteins}

Group III (Figure 4) includes $\mathrm{p} 55 / \mathrm{Gcn} 5$ and three proteins with a single BRD in combination with an Extra-Terminal (ET) Domain. Tt p55/Gcn5 possesses a C-terminal BRD, as does Gcn5 in other organisms. A two-step model was proposed by Taverna and colleagues for yeast Gcn5 which first acetylates H3K14 to provide a platform for binding by its BRD which stimulates its HAT activity on H3K18 (Cieniewicz et al., 2014). By analogy, the role of Tt Gen5 BRD could be similar, stimulating Gcn5 activity after initial recruitment. In this case, the role of the Ibd1 BRD within SAGA would be to recruit SAGA to a region of chromatin perhaps acetylated by the $\mathrm{NuA} 4$ activity described above where it would acetylate $\mathrm{H} 3$, stimulating transcription. Bret-1 Bret-2 and Bret-3 are predicted to have an ET domain. They are similar in domain structure to the BET sub-family of human BRD proteins (BRD2, BRD3, BRD4, and the testis-specific BRDT) that harbor at their aminotermini two BRD followed by an ET domain that mediates protein-protein interactions. BET protein are intense subjects of research in human cells where they are implicated in cancer and are targets for molecules such as JQ1. Differently to human (and yeast) BET that possess two BRDs, Tt BETs only possess one BRD.

\section{HISTONE DEACETYLATION IN Tetrahymena thermophila}

Histone deacetylases remove acetyl groups from lysine residues. Inhibitors that target HDACs have been used to target human diseases such as cancer (Jain and Zain, 2011; Lee et al., 2015). HDAC inhibitors are also being investigated in the treatment of parasitic diseases (Vanagas et al., 2012; Carrillo et al., 2015; Chua et al., 2017). Wiley and colleagues used a bioinformatic query of the Tt MAC genome (Smith et al., 2008) to predict the existence of 18 HDACs that are named THDs (Tetrahymena Histone Deacetylase) and classified according to their similarity to yeast HDACs Rpd3 (class I, 3 members including THD1), Hda1 (class II, 2 members including THD2), and Sir2 (class III, 11 members) with an additional 2 classified as HDAC-like, one of which (Thd5) is predicted to be an ortholog of HDAC11, the smallest HDAC and it is the sole member of HDAC IV family (Gregoretti et al., 2004) implicated in mitosis and meiosis (Sui et al., 2020). Detailed molecular analysis has been performed on Tt HDACs, THD1 (Wiley et al., 2000, 2005; Parker et al., 2007), and THD2 (Smith et al., 2008).

\section{The Class I HDAC THD1}

Class I THD1 was shown to be recruited to developing new macronuclei (Wiley et al., 2000) and to be important for the integrity of macronuclear chromatin in logarithmically dividing cells (Wiley et al., 2005). Cells knocked down for THD1 contain higher amounts of MAC DNA, large extrusion bodies, and enlarged nucleoli (Wiley et al., 2005). It was further shown (Parker et al., 2007) that MAC chromatin in THD1 knockdowns failed to condense during starvation, which was correlated with aberrant hyper-phosphorylation of histone $\mathrm{Hl}$ and the overexpression of $\mathrm{CDC} 2$, encoding the major histone $\mathrm{H} 1$ kinase. Class I HDACs such as Rpd3 are conserved among eukaryotes and are frequently found in corepressor complexes, where they mediate repression by a variety of transcription factors. In humans, the SIN3/RPD3 complex that also contains RbAp46/48 in addition to several other proteins, is targeted to specific genes through protein-protein interactions between SIN3 and either DNA-binding repressors or corepressors (Lewis et al., 2004; Keogh et al., 2005). The metazoan DREAM complex is responsible for the transcriptional regulation of cell cycle-related genes (Sadasivam and DeCaprio, 2013). Recent findings hint at the existence of a DREAM complex in Tt (Zhang et al., 2018). In human cells a Sin3B/HDAC complex robustly interacts with the DREAM complex in a cell-cycle-dependent manner (Bainor et al., 2018). It remains to be determined whether THD1 exerts its phenotype though the Tt DREAM complex.

\section{The Class II HDAC THD2}

Based on work in the 1980s, it was known that although transcription-related acetylation was never observed in the MIC, deposition-related patterns could be observed in the presence of HDAC inhibitors such as sodium butyrate (Allis et al., 1985) indicating that $\mathrm{H} 3$ and $\mathrm{H} 4$ assembled into MIC chromatin were subject to deposition-related acetylation but that was quickly removed post-assembly. GFP-tagging was used to demonstrate that the class II HDAC named Thd2 (Tt histone deacetylase 2) localized specifically to the MIC. A complete deletion of THD2 showed ectopic $\mathrm{H} 3$ and $\mathrm{H} 4$ acetylation in the MIC indicating that WT Thd2 function is to remove deposition related acetylation (Smith et al., 2008). Interestingly, the THD2 KO also displayed a defect in MIC morphology as well as the regulated proteolytic processing of its histone $\mathrm{H} 3$, specifically deficient in producing the fast form of $\mathrm{H} 3$ that in the MIC is phosphorylated on Ser10 (Allis and Gorovsky, 1981; Allis and Wiggins, 1984), a mitotic PTM necessary for chromosome condensation and segregation (Wei et al., 1998, 1999) suggesting that Thd2 functions upstream of the proteolytic cleavage and subsequent phosphorylation of Ser10 on histone H3. It will be interesting to determine if APMS of Thd2 can help identify the elusive protease responsible for this enigmatic process.

\section{Class III HDAC}

Class III histone deacetylases, known as sirtuins, couple the deacetylation of lysine with the hydrolysis of NAD+ by transferring the acetyl group to the ADP-ribose moiety to form O-acetyl-ADP-ribose, releasing free nicotinamide (Dang, 2014). Nicotinamide can thus be used as an inhibitor of sirtuin class HDACs and was used to demonstrate a possible role for the sirtuins in meiotic prophase as well as the degradation of the parental MAC during conjugation (Slade et al., 2011).

More could be learned through the molecular analysis of $\mathrm{Tt}$ HDACs. The use of Trichostatin A, a selective inhibitor of class I and II HDACs, resulted in defects in the progression through meiosis and also affected the deletion of IESs (Duharcourt and Yao, 2002). The identity of relevant HDAC(s) that function in these processes remains unknown. 


\section{CONCLUSION AND PERSPECTIVES}

Tetrahymena thermophila offers a powerful model system with well-developed functional genomics with which to explore and understand the components of the histone acetylation cycle. The unique nuclear biology of $\mathrm{Tt}$ has been extremely useful in the past in the development of the histone acetylation field. Despite its efficacy the model has been underexplored. A complete understanding of the function and mechanism of the $\mathrm{Tt}$ histone acetylation cycle, in particular the role of histone acetylation in the regulation of H2AZ dynamics, should yield fundamental knowledge on the mechanism of transcription. In addition, the position of Tt on the evolutionary tree will permit insight into Alveolate-specific biology such as the composition of NuA4 and the identity of the protist H3K56-specific HAT.

\section{REFERENCES}

Akematsu, T., Fukuda, Y., Garg, J., Fillingham, J. S., Pearlman, R. E., and Loidl, J. (2017). Post-meiotic DNA double-strand breaks occur in Tetrahymena, and require Topoisomerase II and Spo11. eLife 6:e26176.

Allard, S., Utley, R. T., Savard, J., Clarke, A., Grant, P., Brandl, C. J., et al. (1999). $\mathrm{NuA} 4$, an essential transcription adaptor/histone $\mathrm{H} 4$ acetyltransferase complex containing Esalp and the ATM-related cofactor Tralp. EMBO J. 18, 5108-5119. doi: 10.1093/emboj/18.18.5108

Allis, C. D., Allen, R. L., Wiggins, J. C., Chicoine, L. G., and Richman, R. (1984). Proteolytic processing of h1-like histones in chromatin: a physiologically and developmentally regulated event in Tetrahymena micronuclei. J. Cell Biol. 99, 1669-1677. doi: 10.1083/jcb.99.5.1669

Allis, C. D., Berger, S. L., Cote, J., Dent, S., Jenuwien, T., Kouzarides, T., et al. (2007). New nomenclature for chromatin-modifying enzymes. Cell 131, 633-636.

Allis, C. D., Bowen, J. K., Abraham, G. N., Glover, C. V., and Gorovsky, M. A. (1980a). Proteolytic processing of histone H3 in chromatin: a physiologically regulated event in Tetrahymena micronuclei. Cell 20, 55-64. doi: 10.1016/00928674(80)90234- 2

Allis, C. D., Chicoine, L. G., Richman, R., and Schulman, I. G. (1985). Depositionrelated histone acetylation in micronuclei of conjugating Tetrahymena. Proc. Natl. Acad. Sci. U.S.A. 82, 8048-8052. doi: 10.1073/pnas.82.23.8048

Allis, C. D., Glover, C. V., Bowen, J. K., and Gorovsky, M. A. (1980b). Histone variants specific to the transcriptionally active, amitotically dividing macronucleus of the unicellular eucaryote, Tetrahymena thermophila. Cell 20, 609-617. doi: 10.1016/0092-8674(80)90307-4

Allis, C. D., Glover, C. V., and Gorovsky, M. A. (1979). Micronuclei of Tetrahymena contain two types of histone H3. Proc. Natl. Acad. Sci. U.S.A. 76, 4857-4861. doi: $10.1073 /$ pnas.76.10.4857

Allis, C. D., and Gorovsky, M. A. (1981). Histone phosphorylation in macro- and micronuclei of Tetrahymena thermophila. Biochemistry 20, 3828-3833. doi: 10.1021/bi00516a025

Allis, C. D., Richman, R., Gorovsky, M. A., Ziegler, Y. S., Touchstone, B., Bradley, W. A., et al. (1986). hv1 is an evolutionarily conserved H2A variant that is preferentially associated with active genes. J. Biol. Chem. 261, 1941-1948.

Allis, C. D., and Wiggins, J. C. (1984). Proteolytic processing of micronuclear H3 and histone phosphorylation during conjugation in Tetrahymena thermophila. Exp. Cell Res. 153, 287-298. doi: 10.1016/0014-4827(84)90601-3

Allis, C. D., Ziegler, Y. S., Gorovsky, M. A., and Olmsted, J. B. (1982). A conserved histone variant enriched in nucleoli of mammalian cells. Cell 31, 131-136. doi: 10.1016/0092-8674(82)90412-3

Altaf, M., Auger, A., Monnet-Saksouk, J., Brodeur, J., Piquet, S., Cramet, M., et al. (2010). NuA4-dependent acetylation of nucleosomal histones H4 and H2A directly stimulates incorporation of H2A.Z by the SWR1 complex. J. Biol. Chem. 285, 15966-15977. doi: 10.1074/jbc.m110.117069

Andrews, K. T., Tran, T. N., and Fairlie, D. P. (2012). Towards histone deacetylase inhibitors as new antimalarial drugs. Curr. Pharm. Des. 18, 3467-3479.

\section{AUTHOR CONTRIBUTIONS}

SW wrote the manuscript, edited and prepared Figure 3. AS edited the manuscript and prepared Figures 1, 4, SN-S and ND edited the manuscript. JF conceived, wrote, prepared Figure 2, and edited the manuscript. All authors read and approved the final manuscript.

\section{FUNDING}

Research in the Fillingham lab was supported by the Faculty of Science, Ryerson University, in addition to a Discovery Grant from the Natural Sciences and Engineering Research Council of Canada (NSERC).

Arrowsmith, C. H., and Schapira, M. (2019). Targeting non-bromodomain chromatin readers. Nat. Struct. Mol. Biol. 26, 863-869. doi: 10.1038/s41594019-0290-2

Ashraf, K., Nabeel-Shah, S., Garg, J., Saettone, A., Derynck, J., Gingras, A. C., et al. (2019). Proteomic analysis of histones H2A/H2B and variant Hv1 in Tetrahymena thermophila reveals an ancient network of chaperones. Mol. Biol. Evol. 36, 1037-1055. doi: 10.1093/molbev/msz039

Bainor, A. J., Saini, S., Calderon, A., Casado-Polanco, R., Giner-Ramirez, B., Moncada, C., et al. (2018). The HDAC-associated Sin3B protein represses DREAM complex targets and cooperates with APC/C to promote quiescence. Cell. Rep. 25, 2797.e8-2807.e8.

Baker, S. P., and Grant, P. A. (2007). The SAGA continues: expanding the cellular role of a transcriptional co-activator complex. Oncogene 26, 5329-5340. doi: 10.1038/sj.onc. 1210603

Bazan, J. F. (2008). An old HAT in human p300/CBP and yeast Rtt109. Cell Cycle 7, 1884-1886. doi: 10.4161/cc.7.12.6074

Berndsen, C. E., and Denu, J. M. (2008). Catalysis and substrate selection by histone/protein lysine acetyltransferases. Curr. Opin. Struct. Biol. 18, 682-689. doi: 10.1016/j.sbi.2008.11.004

Brahma, S., Udugama, M. I., Kim, J., Hada, A., Bhardwaj, S. K., Hailu, S. G., et al. (2017). INO80 exchanges H2A.Z for H2A by translocating on DNA proximal to histone dimers. Nat. Commun. 8:15616.

Brownell, J. E., and Allis, C. D. (1995). An activity gel assay detects a single, catalytically active histone acetyltransferase subunit in Tetrahymena macronuclei. Proc. Natl. Acad. Sci. U.S.A. 92, 6364-6368. doi: 10.1073/pnas. 92.14.6364

Brownell, J. E., Zhou, J., Ranalli, T., Kobayashi, R., Edmondson, D. G., Roth, S. Y., et al. (1996). Tetrahymena histone acetyltransferase A: a homolog to yeast Gcn5p linking histone acetylation to gene activation. Cell 84, 843-851. doi: 10.1016/s0092-8674(00)81063-6

Burgess, R. J., Zhou, H., Han, J., and Zhang, Z. (2010). A role for Gen5 in replication-coupled nucleosome assembly. Mol. Cell. 37, 469-480. doi: 10.1016/ j.molcel.2010.01.020

Carrillo, A. K., Guiguemde, W. A., and Guy, R. K. (2015). Evaluation of histone deacetylase inhibitors (HDACi) as therapeutic leads for human African trypanosomiasis (HAT). Bioorg. Med. Chem. 23, 5151-5155. doi: 10.1016/j.bmc. 2014.12.066

Cervantes, M. D., Xi, X., Vermaak, D., Yao, M. C., and Malik, H. S. (2006). The CNA1 histone of the ciliate Tetrahymena thermophila is essential for chromosome segregation in the germline micronucleus. Mol. Biol. Cell 17, 485-497. doi: 10.1091/mbc.e05-07-0698

Chalker, D. L., and Yao, M. C. (2001). Nongenic, bidirectional transcription precedes and may promote developmental DNA deletion in Tetrahymena thermophila. Genes Dev. 15, 1287-1298. doi: 10.1101/gad. 884601

Chicoine, L. G., and Allis, C. D. (1986). Regulation of histone acetylation during macronuclear differentiation in Tetrahymena: evidence for control at the level 
of acetylation and deacetylation. Dev. Biol. 116, 477-485. doi: 10.1016/00121606(86)90148-x

Chicoine, L. G., Richman, R., Cook, R. G., Gorovsky, M. A., and Allis, C. D. (1987). A single histone acetyltransferase from Tetrahymena macronuclei catalyzes deposition-related acetylation of free histones and transcriptionrelated acetylation of nucleosomal histones. J. Cell Biol. 105, 127-135. doi: 10.1083/jcb.105.1.127

Chicoine, L. G., Schulman, I. G., Richman, R., Cook, R. G., and Allis, C. D. (1986). Nonrandom utilization of acetylation sites in histones isolated from Tetrahymena. Evidence for functionally distinct $\mathrm{H} 4$ acetylation sites. J. Biol. Chem. 261, 1071-1076.

Chua, M. J., Arnold, M. S., Xu, W., Lancelot, J., Lamotte, S., Spath, G. F., et al. (2017). Effect of clinically approved HDAC inhibitors on Plasmodium, Leishmania and Schistosoma parasite growth. Int. J. Parasitol. Drugs Drug Resist. 7, 42-50. doi: 10.1016/j.ijpddr.2016.12.005

Cieniewicz, A. M., Moreland, L., Ringel, A. E., Mackintosh, S. G., Raman, A., Gilbert, T. M., et al. (2014). The bromodomain of Gen 5 regulates site specificity of lysine acetylation on histone H3. Mol. Cell. Proteomics 13, 2896-2910. doi: 10.1074/mcp.m114.038174

Clapier, C. R., and Cairns, B. R. (2009). The biology of chromatin remodeling complexes. Annu. Rev. Biochem. 78, 273-304.

Cochran, A. G., Conery, A. R., and Sims, R. J. III (2019). Bromodomains: a new target class for drug development. Nat. Rev. Drug Discov. 18, 609-628. doi: 10.1038/s41573-019-0030-7

Collins, S. R., Miller, K. M., Maas, N. L., Roguev, A., Fillingham, J., Chu, C. S., et al. (2007). Functional dissection of protein complexes involved in yeast chromosome biology using a genetic interaction map. Nature 446, 806-810. doi: 10.1038/nature05649

Cui, B., and Gorovsky, M. A. (2006). Centromeric histone H3 is essential for vegetative cell division and for DNA elimination during conjugation in Tetrahymena thermophila. Mol. Cell. Biol. 26, 4499-4510. doi: 10.1128/mcb. 00079-06

Cui, B., Liu, Y., and Gorovsky, M. A. (2006). Deposition and function of histone H3 variants in Tetrahymena thermophila. Mol. Cell. Biol. 26, 7719-7730. doi: 10.1128/mcb.01139-06

Dang, W. (2014). The controversial world of sirtuins. Drug Discov. Today Technol. 12, e9-e17. doi: 10.1016/j.ddtec.2012.08.003

Daniel, J. A., and Grant, P. A. (2007). Multi-tasking on chromatin with the SAGA coactivator complexes. Mutat. Res. 618, 135-148. doi: 10.1016/j.mrfmmm. 2006.09.008

Das, C., Lucia, M. S., Hansen, K. C., and Tyler, J. K. (2009). CBP/p300-mediated acetylation of histone H3 on lysine 56. Nature 459, 113-117. doi: 10.1038/ nature 07861

de Koning, H. (2017). Drug resistance in protozoan parasites. Emerg. Top. Life Sci. 1, 627-632. doi: 10.1042/etls20170113

Dhalluin, C., Carlson, J. E., Zeng, L., He, C., Aggarwal, A. K., and Zhou, M. M. (1999). Structure and ligand of a histone acetyltransferase bromodomain. Nature 399, 491-496. doi: 10.1038/20974

Doyon, Y., and Cote, J. (2004). The highly conserved and multifunctional NuA4 HAT complex. Curr. Opin. Genet. Dev. 14, 147-154. doi: 10.1016/j.gde.2004. 02.009

Draker, R., Ng, M. K., Sarcinella, E., Ignatchenko, V., Kislinger, T., and Cheung, P. (2012). A combination of $\mathrm{H} 2 \mathrm{~A} . \mathrm{Z}$ and $\mathrm{H} 4$ acetylation recruits $\mathrm{Brd} 2$ to chromatin during transcriptional activation. PLoS Genet. 8:e1003047. doi: 10.1371/journal. pgen.1003047

Driscoll, R., Hudson, A., and Jackson, S. P. (2007). Yeast Rtt109 promotes genome stability by acetylating histone H3 on lysine 56. Science $315,649-652$. doi: 10.1126/science. 1135862

Duharcourt, S., and Yao, M. C. (2002). Role of histone deacetylation in developmentally programmed DNA rearrangements in Tetrahymena thermophila. Eukaryot. Cell 1, 293-303. doi: 10.1128/ec.1.2.293-303. 2002

Durant, M., and Pugh, B. F. (2007). NuA4-directed chromatin transactions throughout the Saccharomyces cerevisiae genome. Mol. Cell. Biol. 27, 53275335. doi: 10.1128/mcb.00468-07

Eisen, J. A., Coyne, R. S., Wu, M., Wu, D., Thiagarajan, M., Wortman, J. R., et al. (2006). Macronuclear genome sequence of the ciliate Tetrahymena thermophila, a model eukaryote. PLoS Biol. 4:e286. doi: 10.1371/journal.pbio.0040286
Fan, Q., An, L., and Cui, L. (2004). Plasmodium falciparum histone acetyltransferase, a yeast GCN5 homologue involved in chromatin remodeling. Eukaryot. Cell 3, 264-276. doi: 10.1128/ec.3.2.264-276.2004

Filippakopoulos, P., Picaud, S., Mangos, M., Keates, T., Lambert, J. P., BarsyteLovejoy, D., et al. (2012). Histone recognition and large-scale structural analysis of the human bromodomain family. Cell 149, 214-231. doi: 10.1016/j.cell.2012. 02.013

Fillingham, J., Recht, J., Silva, A. C., Suter, B., Emili, A., Stagljar, I., et al. (2008). Chaperone control of the activity and specificity of the histone $\mathrm{H} 3$ acetyltransferase Rtt109. Mol. Cell. Biol. 28, 4342-4353. doi: 10.1128/mcb. 00182-08

Fillingham, J. S., Garg, J., Tsao, N., Vythilingum, N., Nishikawa, T., and Pearlman, R. E. (2006). Molecular genetic analysis of an SNF2/brahma-related gene in Tetrahymena thermophila suggests roles in growth and nuclear development. Eukaryot. Cell 5, 1347-1359. doi: 10.1128/ec.00149-06

French, C. A., Kutok, J. L., Faquin, W. C., Toretsky, J. A., Antonescu, C. R., Griffin, C. A., et al. (2004). Midline carcinoma of children and young adults with NUT rearrangement. J. Clin. Oncol. 22, 4135-4139. doi: 10.1200/jco.2004.02.107

Fyodorov, D. V., Zhou, B. R., Skoultchi, A. I., and Bai, Y. (2018). Emerging roles of linker histones in regulating chromatin structure and function. Nat. Rev. Mol. Cell Biol. 19, 192-206. doi: 10.1038/nrm.2017.94

Garcia, B. A., Hake, S. B., Diaz, R. L., Kauer, M., Morris, S. A., Recht, J., et al. (2007). Organismal differences in post-translational modifications in histones $\mathrm{H} 3$ and H4. J. Biol. Chem. 282, 7641-7655. doi: 10.1074/jbc.m607900200

Gardner, K. E., Allis, C. D., and Strahl, B. D. (2011). Operating on chromatin, a colorful language where context matters. J. Mol. Biol. 409, 36-46. doi: 10.1016/ j.jmb.2011.01.040

Garg, J., Lambert, J. P., Karsou, A., Marquez, S., Nabeel-Shah, S., Bertucci, V., et al. (2013). Conserved Asf1-importin beta physical interaction in growth and sexual development in the ciliate Tetrahymena thermophila. J. Proteomics 94, 311-326. doi: 10.1016/j.jprot.2013.09.018

Garg, J., Saettone, A., Nabeel-Shah, S., Cadorin, M., Ponce, M., Marquez, S., et al. (2019). The Med31 conserved component of the divergent mediator complex in Tetrahymena thermophila participates in developmental regulation. Curr. Biol. 29, 2371.e-2379.e.

Georgakopoulos, T., and Thireos, G. (1992). Two distinct yeast transcriptional activators require the function of the GCN5 protein to promote normal levels of transcription. EMBO J. 11, 4145-4152. doi: 10.1002/j.1460-2075.1992.tb05507. $\mathrm{x}$

Grant, P. A., Duggan, L., Cote, J., Roberts, S. M., Brownell, J. E., Candau, R., et al. (1997). Yeast Gcn5 functions in two multisubunit complexes to acetylate nucleosomal histones: characterization of an Ada complex and the SAGA (Spt/Ada) complex. Genes Dev. 11, 1640-1650. doi: 10.1101/gad.11.13.1640

Grant, P. A., Eberharter, A., John, S., Cook, R. G., Turner, B. M., and Workman, J. L. (1999). Expanded lysine acetylation specificity of Gcn5 in native complexes. J. Biol. Chem. 274, 5895-5900. doi: 10.1074/jbc.274.9.5895

Grant, P. A., Schieltz, D., Pray-Grant, M. G., Steger, D. J., Reese, J. C., Yates, J. R. III, et al. (1998). A subset of TAF(II)s are integral components of the SAGA complex required for nucleosome acetylation and transcriptional stimulation. Cell 94, 45-53. doi: 10.1016/s0092-8674(00)81220-9

Gregoretti, I. V., Lee, Y. M., and Goodson, H. V. (2004). Molecular evolution of the histone deacetylase family: functional implications of phylogenetic analysis. J. Mol. Biol. 338, 17-31. doi: 10.1016/j.jmb.2004.02.006

Grunstein, M., and Allis, C. D. (2018). Genetics, biochemistry, and "Simple" organisms converge to unlock secrets in histone biology: the 2018 albert lasker basic medical research award. JAMA 320, 1233-1234.

Gupta, D. K., Patra, A. T., Zhu, L., Gupta, A. P., and Bozdech, Z. (2016). DNA damage regulation and its role in drug-related phenotypes in the malaria parasites. Sci. Rep. 6:23603.

Hamilton, E. P., Kapusta, A., Huvos, P. E., Bidwell, S. L., Zafar, N., Tang, H., et al. (2016). Structure of the germline genome of Tetrahymena thermophila and relationship to the massively rearranged somatic genome. eLife 5:e19090.

Hanquier, J., Gimeno, T., Jeffers, V., and Sullivan, W. J. Jr. (2020). Evaluating the GCN5b bromodomain as a novel therapeutic target against the parasite Toxoplasma gondii. Exp. Parasitol. 211:107868. doi: 10.1016/j.exppara.2020. 107868

Henikoff, S., and Smith, M. M. (2015). Histone variants and epigenetics. Cold Spring Harb. Perspect. Biol. 7:a019364. doi: 10.1101/cshperspect.a019364 
Hoeijmakers, W. A. M., Miao, J., Schmidt, S., Toenhake, C. G., Shrestha, S., Venhuizen, J., et al. (2019). Epigenetic reader complexes of the human malaria parasite, Plasmodium falciparum. Nucleic Acids Res. 47, 11574-11588. doi: 10.1093/nar/gkz1044

Jacquet, K., Fradet-Turcotte, A., Avvakumov, N., Lambert, J. P., Roques, C., Pandita, R. K., et al. (2016). The TIP60 complex regulates bivalent chromatin recognition by 53BP1 through Direct H4K20me binding and H2AK15 acetylation. Mol. Cell. 62, 409-421. doi: 10.1016/j.molcel.2016.03.031

Jain, A. K., and Barton, M. C. (2017). Bromodomain histone readers and cancer. J. Mol. Biol. 429, 2003-2010. doi: 10.1016/j.jmb.2016.11.020

Jain, S., and Zain, J. (2011). Romidepsin in the treatment of cutaneous T-cell lymphoma. J. Blood Med. 2, 37-47.

Jeffers, V., Gao, H., Checkley, L. A., Liu, Y., Ferdig, M. T., and Sullivan, W. J. Jr. (2016). Garcinol Inhibits GCN5-mediated lysine acetyltransferase activity and prevents replication of the parasite Toxoplasma gondii. Antimicrob. Agents Chemother. 60, 2164-2170. doi: 10.1128/aac.03059-15

Jeffers, V., and Sullivan, W. J. Jr. (2012). Lysine acetylation is widespread on proteins of diverse function and localization in the protozoan parasite Toxoplasma gondii. Eukaryot. Cell 11, 735-742. doi: 10.1128/ec.00088-12

Jeffers, V., Yang, C., Huang, S., and Sullivan, W. J. Jr. (2017). Bromodomains in protozoan parasites: evolution, function, and opportunities for drug development. Microbiol. Mol. Biol. Rev. 81:e00047-16.

Johnson, C. N., Adkins, N. L., and Georgel, P. (2005). Chromatin remodeling complexes: ATP-dependent machines in action. Biochem. Cell Biol. 83, 405-417. doi: 10.1139/o05-115

Josling, G. A., Petter, M., Oehring, S. C., Gupta, A. P., Dietz, O., Wilson, D. W., et al. (2015). A plasmodium falciparum bromodomain protein regulates invasion gene expression. Cell Host Microbe 17, 741-751. doi: 10.1016/j.chom.2015.05. 009

Kawahara, T., Siegel, T. N., Ingram, A. K., Alsford, S., Cross, G. A., and Horn, D. (2008). Two essential MYST-family proteins display distinct roles in histone H4K10 acetylation and telomeric silencing in trypanosomes. Mol. Microbiol. 69, 1054-1068. doi: 10.1111/j.1365-2958.2008.06346.x

Keogh, M. C., Kurdistani, S. K., Morris, S. A., Ahn, S. H., Podolny, V., Collins, S. R., et al. (2005). Cotranscriptional set 2 methylation of histone H3 lysine 36 recruits a repressive Rpd3 complex. Cell 123, 593-605. doi: 10.1016/j.cell.2005.10.025

Kobor, M. S., Venkatasubrahmanyam, S., Meneghini, M. D., Gin, J. W., Jennings, J. L., Link, A. J., et al. (2004). A protein complex containing the conserved Swi2/Snf2-related ATPase Swr1p deposits histone variant H2A.Z into euchromatin. PLoS Biol. 2:E131. doi: 10.1371/journal.pbio.0020131

Kougnassoukou Tchara, P. E., Filippakopoulos, P., and Lambert, J. P. (2019). Emerging tools to investigate bromodomain functions. Methods doi: 10.1016/ j.ymeth.2019.11.003 [Epub ahead of print].

Kuo, M. H., and Allis, C. D. (1998). Roles of histone acetyltransferases and deacetylases in gene regulation. Bioessays 20, 615-626. doi: 10.1002/(sici)15211878(199808)20:8<615::aid-bies4>3.0.co;2-h

Lalonde, M. E., Cheng, X., and Cote, J. (2014). Histone target selection within chromatin: an exemplary case of teamwork. Genes Dev. 28, 1029-1041. doi: 10.1101/gad.236331.113

Lee, H. Z., Kwitkowski, V. E., Del Valle, P. L., Ricci, M. S., Saber, H., Habtemariam, B. A., et al. (2015). FDA approval: belinostat for the treatment of patients with relapsed or refractory peripheral T-cell lymphoma. Clin. Cancer Res. 21, 2666-2670. doi: 10.1158/1078-0432.ccr-14-3119

Lewis, P. W., Beall, E. L., Fleischer, T. C., Georlette, D., Link, A. J., and Botchan, M. R. (2004). Identification of a Drosophila Myb-E2F2/RBF transcriptional repressor complex. Genes Dev. 18, 2929-2940. doi: 10.1101/gad.1255204

Li, Y., Li, H., Sui, M., Li, M., Wang, J., Meng, Y., et al. (2019). Fungal acetylome comparative analysis identifies an essential role of acetylation in human fungal pathogen virulence. Commun. Biol. 2:154.

Li, Y., Wen, H., Xi, Y., Tanaka, K., Wang, H., Peng, D., et al. (2014). AF9 YEATS domain links histone acetylation to DOT1L-mediated H3K79 methylation. Cell 159, 558-571. doi: 10.1016/j.cell.2014.09.049

Lin, R., Leone, J. W., Cook, R. G., and Allis, C. D. (1989). Antibodies specific to acetylated histones document the existence of deposition- and transcriptionrelated histone acetylation in Tetrahymena. J. Cell Biol. 108, 1577-1588. doi: 10.1083/jcb.108.5.1577

Liu, Y., Taverna, S. D., Muratore, T. L., Shabanowitz, J., Hunt, D. F., and Allis, C. D. (2007). RNAi-dependent H3K27 methylation is required for heterochromatin formation and DNA elimination in Tetrahymena. Genes Dev. 21, 1530-1545. doi: $10.1101 / \operatorname{gad} .1544207$

Luger, K., Mader, A. W., Richmond, R. K., Sargent, D. F., and Richmond, T. J. (1997). Crystal structure of the nucleosome core particle at 2.8 A resolution. Nature 389, 251-260.

Martindale, D. W., Allis, C. D., and Bruns, P. J. (1982). Conjugation in Tetrahymena thermophila. A temporal analysis of cytological stages. Exp. Cell Res. 140, 227-236. doi: 10.1016/0014-4827(82)90172-0

Martindale, D. W., Allis, C. D., and Bruns, P. J. (1985). RNA and protein synthesis during meiotic prophase in Tetrahymena thermophila. J. Protozool. 32, 644-649. doi: 10.1111/j.1550-7408.1985.tb03094.x

Masumoto, H., Hawke, D., Kobayashi, R., and Verreault, A. (2005). A role for cellcycle-regulated histone $\mathrm{H} 3$ lysine 56 acetylation in the DNA damage response. Nature 436, 294-298. doi: 10.1038/nature03714

Miao, J., Fan, Q., Cui, L., Li, X., Wang, H., Ning, G., et al. (2010). The MYST family histone acetyltransferase regulates gene expression and cell cycle in malaria parasite Plasmodium falciparum. Mol. Microbiol. 78, 883-902. doi: 10.1111/ j.1365-2958.2010.07371.x

Miao, J., Lawrence, M., Jeffers, V., Zhao, F., Parker, D., Ge, Y., et al. (2013). Extensive lysine acetylation occurs in evolutionarily conserved metabolic pathways and parasite-specific functions during Plasmodium falciparum intraerythrocytic development. Mol. Microbiol. 89, 660-675. doi: 10.1111/mmi. 12303

Miao, W., Xiong, J., Bowen, J., Wang, W., Liu, Y., Braguinets, O., et al. (2009). Microarray analyses of gene expression during the Tetrahymena thermophila life cycle. PLoS One 4:e4429. doi: 10.1371/journal.pone.0004429

Morrison, A. J., and Shen, X. (2009). Chromatin remodelling beyond transcription: the INO80 and SWR1 complexes. Nat. Rev. Mol. Cell Biol. 10, 373-384. doi: $10.1038 / \mathrm{nrm} 2693$

Nabeel-Shah, S., Ashraf, K., Saettone, A., Garg, J., Derynck, J., Lambert, J. P., et al. (2020). Nucleus-specific linker histones Hhol and Mlh1 form distinct protein interactions during growth, starvation and development in Tetrahymena thermophila. Sci. Rep. 10:168.

Nagarajan, P., Ge, Z., Sirbu, B., Doughty, C., Agudelo Garcia, P. A., Schlederer, M., et al. (2013). Histone acetyl transferase 1 is essential for mammalian development, genome stability, and the processing of newly synthesized histones H3 and H4. PLoS Genet. 9:e1003518. doi: 10.1371/journal.pgen. 1003518

Nguyen, H. H. T., Yeoh, L. M., Chisholm, S. A., and Duffy, M. F. (2020). Developments in drug design strategies for bromodomain protein inhibitors to target Plasmodium falciparum parasites. Expert Opin. Drug Discov. 15, 415-425. doi: 10.1080/17460441.2020.1704251

No Author (2018). Chasing histone biology from sea urchins to yeast. Cell 175, 27-29. doi: 10.1016/j.cell.2018.08.008

Ohba, R., Steger, D. J., Brownell, J. E., Mizzen, C. A., Cook, R. G., Cote, J., et al. (1999). A novel H2A/H4 nucleosomal histone acetyltransferase in Tetrahymena thermophila. Mol. Cell. Biol. 19, 2061-2068. doi: 10.1128/mcb.19.3.2061

Orias, E., Cervantes, M. D., and Hamilton, E. P. (2011). Tetrahymena thermophila, a unicellular eukaryote with separate germline and somatic genomes. Res. Microbiol. 162, 578-586. doi: 10.1016/j.resmic.2011.05.001

Orias, E., and Flacks, M. (1975). Macronuclear genetics of Tetrahymena. I. Random distribution of macronuclear genecopies in pyriformis, T., syngen 1. Genetics 79, 187-206.

Papamichos-Chronakis, M., Watanabe, S., Rando, O. J., and Peterson, C. L. (2011). Global regulation of H2A.Z localization by the INO80 chromatin-remodeling enzyme is essential for genome integrity. Cell 144, 200-213. doi: 10.1016/j.cell. 2010.12.021

Parker, K., Maxson, J., Mooney, A., and Wiley, E. A. (2007). Class I histone deacetylase Thd $1 \mathrm{p}$ promotes global chromatin condensation in Tetrahymena thermophila. Eukaryot. Cell 6, 1913-1924. doi: 10.1128/ec.00217-07

Parthun, M. R. (2007). Hat1: the emerging cellular roles of a type B histone acetyltransferase. Oncogene 26, 5319-5328. doi: 10.1038/sj.onc.1 210602

Parthun, M. R. (2013). Histone acetyltransferase 1: more than just an enzyme? Biochim. Biophys. Acta 1819, 256-263. doi: 10.1016/j.bbagrm.2011.07.006

Parthun, M. R., Widom, J., and Gottschling, D. E. (1996). The major cytoplasmic histone acetyltransferase in yeast: links to chromatin replication and histone metabolism. Cell 87, 85-94. doi: 10.1016/s0092-8674(00)81325-2 
Poli, J., Gasser, S. M., and Papamichos-Chronakis, M. (2017). The INO80 remodeller in transcription, replication and repair. Philos. Trans. R. Soc. Lond. B Biol. Sci. 372:20160290. doi: 10.1098/rstb.2016.0290

Recht, J., Tsubota, T., Tanny, J. C., Diaz, R. L., Berger, J. M., Zhang, X., et al. (2006). Histone chaperone Asf1 is required for histone H3 lysine 56 acetylation, a modification associated with S phase in mitosis and meiosis. Proc. Natl. Acad. Sci. U.S.A. 103, 6988-6993. doi: 10.1073/pnas. 0601676103

Ren, Q., and Gorovsky, M. A. (2001). Histone H2A.Z acetylation modulates an essential charge patch. Mol. Cell. 7, 1329-1335. doi: 10.1016/s1097-2765(01) 00269-6

Richman, R., Chicoine, L. G., Collini, M. P., Cook, R. G., and Allis, C. D. (1988). Micronuclei and the cytoplasm of growing Tetrahymena contain a histone acetylase activity which is highly specific for free histone H4. J. Cell Biol. 106, 1017-1026. doi: 10.1083/jcb.106.4.1017

Richters, A., and Koehler, A. N. (2017). Epigenetic modulation using small molecules - targeting histone acetyltransferases in disease. Curr. Med. Chem. 24, 4121-4150.

Ruhl, D. D., Jin, J., Cai, Y., Swanson, S., Florens, L., Washburn, M. P., et al. (2006). Purification of a human SRCAP complex that remodels chromatin by incorporating the histone variant H2A.Z into nucleosomes. Biochemistry 45, 5671-5677. doi: 10.1021/bi060043d

Sadasivam, S., and DeCaprio, J. A. (2013). The DREAM complex: master coordinator of cell cycle-dependent gene expression. Nat. Rev. Cancer 13, 585-595. doi: $10.1038 / \mathrm{nrc} 3556$

Saettone, A., Garg, J., Lambert, J. P., Nabeel-Shah, S., Ponce, M., Burtch, A., et al. (2018). The bromodomain-containing protein Ibd1 links multiple chromatin-related protein complexes to highly expressed genes in Tetrahymena thermophila. Epigenet. Chromatin 11:10.

Saettone, A., Nabeel-Shah, S., Garg, J., Lambert, J. P., Pearlman, R. E., and Fillingham, J. (2019a). Functional proteomics of nuclear proteins in Tetrahymena thermophila: a review. Genes 10:333. doi: 10.3390/genes10050333

Saettone, A., Ponce, M., Nabeel-Shah, S., and Fillingham, J. (2019b). RACS: rapid analysis of ChIP-Seq data for contig based genomes. BMC Bioinformatics 20:533. doi: 10.1186/s12859-019-3100-2

Saha, A., Wittmeyer, J., and Cairns, B. R. (2006). Chromatin remodelling: the industrial revolution of DNA around histones. Nat. Rev. Mol. Cell Biol. 7, 437-447. doi: 10.1038/nrm1945

Santos, J. M., Josling, G., Ross, P., Joshi, P., Orchard, L., Campbell, T., et al. (2017). Red blood cell invasion by the malaria parasite is coordinated by the PfAP2-I transcription factor. Cell Host Microbe 21:e10.

Schulz, D., Mugnier, M. R., Paulsen, E. M., Kim, H. S., Chung, C. W., Tough, D. F., et al. (2015). Bromodomain proteins contribute to maintenance of bloodstream form stage identity in the African Trypanosome. PLoS Biol. 13:e1002316. doi: 10.1371/journal.pbio.1002316

Shen, X., Yu, L., Weir, J. W., and Gorovsky, M. A. (1995). Linker histones are not essential and affect chromatin condensation in vivo. Cell 82, 47-56. doi: 10.1016/0092-8674(95)90051-9

Shilatifard, A. (2012). The COMPASS family of histone H3K4 methylases: mechanisms of regulation in development and disease pathogenesis. Annu. Rev. Biochem. 81, 65-95. doi: 10.1146/annurev-biochem-051710-134100

Slade, K. M., Freggiaro, S., Cottrell, K. A., Smith, J. J., and Wiley, E. A. (2011). Sirtuin-mediated nuclear differentiation and programmed degradation in Tetrahymena. BMC Cell Biol. 12:40. doi: 10.1186/1471-2121-12-40

Smith, J. J., Torigoe, S. E., Maxson, J., Fish, L. C., and Wiley, E. A. (2008). A class II histone deacetylase acts on newly synthesized histones in Tetrahymena. Eukaryot. Cell 7, 471-482. doi: 10.1128/ec.00409-07

Sobel, R. E., Cook, R. G., Perry, C. A., Annunziato, A. T., and Allis, C. D. (1995). Conservation of deposition-related acetylation sites in newly synthesized histones H3 and H4. Proc. Natl. Acad. Sci. U.S.A. 92, 1237-1241. doi: 10.1073/ pnas.92.4.1237

Somech, R., Izraeli, S., and Simson, A. J. (2004). Histone deacetylase inhibitors-a new tool to treat cancer. Cancer Treat. Rev. 30, 461-472. doi: 10.1016/j.ctrv. 2004.04.006

Stargell, L. A., Bowen, J., Dadd, C. A., Dedon, P. C., Davis, M., Cook, R. G., et al. (1993). Temporal and spatial association of histone H2A variant hv1 with transcriptionally competent chromatin during nuclear development in Tetrahymena thermophila. Genes Dev. 7, 2641-2651. doi: 10.1101/gad.7.12b. 2641
Stover, N. A., Krieger, C. J., Binkley, G., Dong, Q., Fisk, D. G., Nash, R., et al. (2006). Tetrahymena genome database (TGD): a new genomic resource for Tetrahymena thermophila research. Nucleic Acids Res. 34, D500-D503.

Sui, L., Zhang, S., Huang, R., and Li, Z. (2020). HDAC11 promotes meiotic apparatus assembly during mouse oocyte maturation via decreasing H4K16 and alpha-tubulin acetylation. Cell Cycle 19, 354-362. doi: 10.1080/15384101.2019. 1711315

Taverna, S. D., Coyne, R. S., and Allis, C. D. (2002). Methylation of histone h3 at lysine 9 targets programmed DNA elimination in Tetrahymena. Cell 110, 701-711. doi: 10.1016/s0092-8674(02)00941-8

Taverna, S. D., Ueberheide, B. M., Liu, Y., Tackett, A. J., Diaz, R. L., Shabanowitz, J., et al. (2007). Long-distance combinatorial linkage between methylation and acetylation on histone H3 N termini. Proc. Natl. Acad. Sci. U.S.A. 104, 2086-2091. doi: 10.1073/pnas.0610993104

Teo, G., Liu, G., Zhang, J., Nesvizhskii, A. I., Gingras, A. C., and Choi, H. (2014). SAINTexpress: improvements and additional features in Significance Analysis of INTeractome software. J. Proteomics 100, 37-43. doi: 10.1016/j.jprot.2013. 10.023

Thatcher, T. H., MacGaffey, J., Bowen, J., Horowitz, S., Shapiro, D. L., and Gorovsky, M. A. (1994). Independent evolutionary origin of histone H3.3-like variants of animals and Tetrahymena. Nucleic Acids Res. 22, 180-186. doi: 10.1093/nar/22.2.180

Tjeertes, J. V., Miller, K. M., and Jackson, S. P. (2009). Screen for DNA-damageresponsive histone modifications identifies $\mathrm{H} 3 \mathrm{~K} 9 \mathrm{Ac}$ and $\mathrm{H} 3 \mathrm{~K} 56 \mathrm{Ac}$ in human cells. EMBO J. 28, 1878-1889. doi: 10.1038/emboj.2009.119

Travis, G. H., Colavito-Shepanski, M., and Grunstein, M. (1984). Extensive purification and characterization of chromatin-bound histone acetyltransferase from Saccharomyces cerevisiae. J. Biol. Chem. 259, 14406-14412.

Vanagas, L., Jeffers, V., Bogado, S. S., Dalmasso, M. C., Sullivan, W. J. Jr., and Angel, S. O. (2012). Toxoplasma histone acetylation remodelers as novel drug targets. Expert Rev. Anti Infect. Ther. 10, 1189-1201. doi: 10.1586/eri. 12.100

Vavra, K. J., Allis, C. D., and Gorovsky, M. A. (1982a). Regulation of histone acetylation in Tetrahymena macro- and micronuclei. J. Biol. Chem. 257, 25912598.

Vavra, K. J., Colavito-Shepanski, M., and Gorovsky, M. A. (1982b). Histone acetylation and the deoxyribonuclease I sensitivity of the Tetrahymena ribosomal gene. Biochemistry 21, 1772-1781. doi: 10.1021/bi00537a012

Verreault, A., Kaufman, P. D., Kobayashi, R., and Stillman, B. (1998). Nucleosomal DNA regulates the core-histone-binding subunit of the human Hat1 acetyltransferase. Curr. Biol. 8, 96-108. doi: 10.1016/s0960-9822(98) 70040-5

Wai, D. C. C., Szyszka, T. N., Campbell, A. E., Kwong, C., Wilkinson-White, L. E., Silva, A. P. G., et al. (2018). The BRD3 ET domain recognizes a short peptide motif through a mechanism that is conserved across chromatin remodelers and transcriptional regulators. J. Biol. Chem. 293, 7160-7175. doi: 10.1074/jbc. ra117.000678

Wang, J., Dixon, S. E., Ting, L. M., Liu, T. K., Jeffers, V., Croken, M. M., et al. (2014). Lysine acetyltransferase GCN5b interacts with AP2 factors and is required for Toxoplasma gondii proliferation. PLoS Pathog. 10:e1003830. doi: 10.1371/ journal.ppat. 1003830

Wang, J., Gao, S., Mostovoy, Y., Kang, Y., Zagoskin, M., Sun, Y., et al. (2017). Comparative genome analysis of programmed DNA elimination in nematodes. Genome Res. 27, 2001-2014. doi: 10.1101/gr.225730.117

Wang, X., Wang, S., Troisi, E. C., Howard, T. P., Haswell, J. R., Wolf, B. K., et al. (2019). BRD9 defines a SWI/SNF sub-complex and constitutes a specific vulnerability in malignant rhabdoid tumors. Nat. Commun. 10:1881.

Wei, Y., Mizzen, C. A., Cook, R. G., Gorovsky, M. A., and Allis, C. D. (1998). Phosphorylation of histone $\mathrm{H} 3$ at serine 10 is correlated with chromosome condensation during mitosis and meiosis in Tetrahymena. Proc. Natl. Acad. Sci. U.S.A. 95, 7480-7484. doi: 10.1073/pnas.95.13.7480

Wei, Y., Yu, L., Bowen, J., Gorovsky, M. A., and Allis, C. D. (1999). Phosphorylation of histone $\mathrm{H} 3$ is required for proper chromosome condensation and segregation. Cell 97, 99-109. doi: 10.1016/s0092-8674(00)80718-7

Wenkert, D., and Allis, C. D. (1984). Timing of the appearance of macronuclearspecific histone variant hv1 and gene expression in developing new macronuclei of Tetrahymena thermophila. J. Cell Biol. 98, 2107-2117. doi: 10.1083/jcb.98.6. 2107 
Wiley, E. A., Myers, T., Parker, K., Braun, T., and Yao, M. C. (2005). Class I histone deacetylase Thd1p affects nuclear integrity in Tetrahymena thermophila. Eukaryot. Cell 4, 981-990. doi: 10.1128/ec.4.5.981-990. 2005

Wiley, E. A., Ohba, R., Yao, M. C., and Allis, C. D. (2000). Developmentally regulated $\mathrm{rpd} 3 \mathrm{p}$ homolog specific to the transcriptionally active macronucleus of vegetative Tetrahymena thermophila. Mol. Cell. Biol. 20, 8319-8328. doi: 10.1128/mcb.20.22.8319-8328.2000

World Health Organization [WHO] (2010). Working to Overcome the Global Impact of Neglected Tropical Diseases: First WHO Report on Neglected Tropical Diseases. Geneva: World Health Organization.

Wurtele, H., Tsao, S., Lepine, G., Mullick, A., Tremblay, J., Drogaris, P., et al. (2010). Modulation of histone $\mathrm{H} 3$ lysine 56 acetylation as an antifungal therapeutic strategy. Nat. Med. 16, 774-780. doi: 10.1038/nm.2175

Xiong, J., Yuan, D., Fillingham, J. S., Garg, J., Lu, X., Chang, Y., et al. (2011). Gene network landscape of the ciliate Tetrahymena thermophila. PLoS One 6:e20124. doi: 10.1371/journal.pone.0020124

Yan, L., Wang, L., Tian, Y., Xia, X., and Chen, Z. (2016). Structure and regulation of the chromatin remodeller ISWI. Nature 540, 466-469. doi: 10.1038/ nature 20590

Yang, X., Wu, X., Zhang, J., Zhang, X., Xu, C., Liao, S., et al. (2017). Recognition of hyperacetylated N-terminus of $\mathrm{H} 2 \mathrm{AZ}$ by TbBDF2 from Trypanosoma brucei. Biochem. J. 474, 3817-3830. doi: 10.1042/bcj20170619

Yao, M., Chao, J., and Cheng, C. (2015). "Programmed genome rearrangements in Tetrahymena," in Mobile DNA III, eds N. Craig, M. Chandler, M. Gellert, A. Lambowitz, P. Rice, and S. Sandmeyer (Washington, DC: ASM Press), 349-367. doi: 10.1128/microbiolspec.MDNA3-0012-2014
Yu, L., and Gorovsky, M. A. (1997). Constitutive expression, not a particular primary sequence, is the important feature of the $\mathrm{H} 3$ replacement variant hv2 in Tetrahymena thermophila. Mol. Cell. Biol. 17, 6303-6310. doi: 10.1128/mcb. 17.11.6303

Zaware, N., and Zhou, M. M. (2019). Bromodomain biology and drug discovery. Nat. Struct. Mol. Biol. 26, 870-879. doi: 10.1038/s41594-019-0309-8

Zeng, L., Zhang, Q., Li, S., Plotnikov, A. N., Walsh, M. J., and Zhou, M. M. (2010). Mechanism and regulation of acetylated histone binding by the tandem PHD finger of DPF3b. Nature 466, 258-262. doi: 10.1038/nature09139

Zhang, J., Yan, G., Tian, M., Ma, Y., Xiong, J., and Miao, W. (2018). A DPlike transcription factor protein interacts with E2fll to regulate meiosis in Tetrahymena thermophila. Cell Cycle 17, 634-642. doi: 10.1080/15384101.2018. 1431595

Zhao, S., Xu, W., Jiang, W., Yu, W., Lin, Y., Zhang, T., et al. (2010). Regulation of cellular metabolism by protein lysine acetylation. Science 327, 1000-1004.

Conflict of Interest: The authors declare that the research was conducted in the absence of any commercial or financial relationships that could be construed as a potential conflict of interest.

Copyright (c) 2020 Wahab, Saettone, Nabeel-Shah, Dannah and Fillingham. This is an open-access article distributed under the terms of the Creative Commons Attribution License (CC BY). The use, distribution or reproduction in other forums is permitted, provided the original author(s) and the copyright owner(s) are credited and that the original publication in this journal is cited, in accordance with accepted academic practice. No use, distribution or reproduction is permitted which does not comply with these terms. 